\title{
Dielectric tuning and coupling of whispering gallery modes using an anisotropic prism
}

\author{
Matthew R. Foreman ${ }^{1,}$, Florian Sedlmeir ${ }^{1}$, Harald G. L. Schwefel ${ }^{2}$, And Gerd Leuchs ${ }^{1,3}$ \\ ${ }^{1}$ Max Planck Institute for the Science of Light, Günther-Scharowsky-Straße 1, 91058 Erlangen, Germany \\ ${ }^{2}$ Department of Physics, University of Otago, Dunedin, New Zealand \\ ${ }^{3}$ Institut für Optik, Information und Photonik, Universität Erlangen-Nürnberg, Staudtstraße 7/B2, 91058 Erlangen, Germany \\ *Corresponding author: matthew.foreman@mpl.mpg.de
}

Compiled August 25, 2016

Optical whispering gallery mode (WGM) resonators are a powerful and versatile tool used in many branches of science. Fine tuning of the central frequency and line width of individual resonances is however desirable in a number of applications including frequency conversion, optical communications and efficient light-matter coupling. To this end we present a detailed theoretical analysis of dielectric tuning of WGMs supported in axisymmetric resonators. Using the Bethe-Schwinger equation and adopting an angular spectrum field representation we study the resonance shift and mode broadening of high $Q$ WGMs when a planar dielectric substrate is brought close to the resonator. Particular focus is given to use of a uniaxial substrate with an arbitrarily aligned optic axis. Competing red and blue resonance shifts ( $30 \mathrm{MHz}$ ), deriving from generation of a near field material polarisation and back action from the radiation continuum respectively, are found. Anomalous resonance shifts can hence be observed depending on the substrate material, whereas mode broadening on the order of $\sim 50 \mathrm{MHz}$ can also be realised. Furthermore, polarisation-selective coupling with extinction ratios of $>10^{4}$ can be achieved when the resonator and substrate are of the same composition and their optic axes are chosen correctly. Double refraction and properties of out-coupled beams are also discussed. (๑) 2016 Optical Society of America

OCIS codes: (140.4780) Optical resonators, (260.5740) Resonance, (290.5825) Scattering theory, (160.1190) Anisotropic optical materials

\section{INTRODUCTION}

Whispering gallery mode (WGM) resonators are a powerful and versatile tool in modern day optics and have found great employ as novel light sources [1-4], in spectroscopic studies [5], frequency comb generation $[6,7]$, quantum electrodynamics $[8,9]$, sensing [10], nonlinear optics [11, 12] and optomechanics [13, 14]. Such extensive usage derives from the narrow bandwidths, high field strengths and small modal volumes boasted by WGMs. Primarily, the resonance structure of WGM resonators is dictated by their geometry and material composition, however, tunability of resonance properties, such as central frequency and line width, is desirable in a number of applications. For example, WGM microcavities can be used as tunable filters or switches, which play a central role in optical signal processing and classical communication networks [15]. Alternatively, matching the frequency and bandwidth of WGMs to those of atomic transitions [16] enables efficient light-matter coupling required in quantum communications [17] and information processing [18]. Controlled coupling and tuning of WGM resonances can, moreover, greatly improve the efficiency of polarisation conversion [19] and nonlinear optical processes such as second harmonic generation or parametric oscillation $[20,21]$ and is thus important for realisation of novel light sources and frequency converters.

A number of strategies for tuning WGM resonances based on: external temperature control [9]; electro-optical [22] or thermooptical effects [23-25]; and variation of an applied strain [26-28] or pressure $[29,30]$, have previously been reported in the literature. So-called dielectric tuning, in which a dielectric substrate or prism (note we shall use the terms prism and substrate interchangeably throughout this text) is brought into close proximity to a WGM resonator, has also been proposed and demonstrated as a route to continuous fine tuning $[16,31]$. Several numerical and approximate analytic techniques have been developed to study the properties of WGMs in the presence of local dielectric or plasmonic perturbations [32-35] and to model cavitywaveguide coupling [36-40]. To date, however, limited consideration has been given to birefringent resonators or couplers which are attracting increasing attention, especially in nonlinear optics 
$[12,41]$. In this work, we present a detailed theoretical formalism describing dielectric frequency tuning of WGM resonances using planar substrates, in addition to quantifying substrate induced line width changes. Emphasis is placed on the use of arbitrary uniaxial dielectric planar substrates, which we show can enable differential tuning and selective out-coupling of transverse electric (TE) and transverse magnetic (TM) WGMs [42]. We eschew a numerical approach in favour of an analytic treatment due to the fundamental insights afforded by the latter, such as identifying the origin of possible anomalous blue shifts. Such analytic approaches are also frequently simpler to implement and facilitate faster computations. The structure of this article is therefore as follows. In Section 2 we first generalise the analytic WGM profiles given in [43] to account for the open nature of WGM resonators and detail the properties of WGMs in an isolated uniaxial axisymmetric resonator required in subsequent derivations. After establishing a suitable angular spectrum representation of WGMs in Section 3 we continue to determine the interface induced coupling and resulting mode distributions in the presence of a uniaxial dielectric prism. Resonance shifts and mode broadening induced by introduction of a planar substrate are subsequently derived by exploiting the Bethe-Schwinger cavity perturbation formula in Section 4 . Critically, far-field contributions, which can give rise to anomalous radiative shifts in open resonators [44], are incorporated into the Bethe-Schwinger equation. Section 5 proceeds to present a number of illustrative numerical results, before we finally conclude in Section 6 .

\section{WHISPERING GALLERY MODES IN OPEN AXISYM- METRIC RESONATORS}

In this section we discuss a number of WGM properties that will be required in later sections. We consider a WGM with vacuum wavelength $\lambda=2 \pi / k$ supported in a uniaxial axisymmetric resonator in air with major radius $R$ and for which the radius of curvature of the outer surface in the polar direction (rim radius) is $P$ (see Figure $1(\mathrm{a})$ ). We denote the ordinary and extraordinary refractive index of the resonator by $n_{o}$ and $n_{e}$ respectively. Throughout this work we assume that $R \gg \lambda$ and $P \gg \lambda$ and that the optic axis of the resonator is parallel to the symmetry axis (z-cut). Furthermore, we restrict attention to WGMs that are localised in the equatorial plane and lie close to the resonator surface such that we can use the toroidal coordinates $(\rho, \theta, \phi)$, where $\rho=|\rho|$ is the distance from the centre of curvature of the resonator rim, $\theta$ is the toroidal polar angle and $\phi$ is the azimuthal angle (see Figure 1). Due to the strong confinement of WGMs, this local coordinate system is also suitable for describing disk-like, ellipsoidal and spherical resonators [43, 45]. With these assumptions the amplitude of the dominant electric field component is well described using a scalar Helmholtz equation [43]. WGMs, however, are either TE or TM polarised i.e. the corresponding electric- and magnetic-field vectors lie tangential to the resonator surface. Within the resonator the associated unit (electric field) polarisation vectors can thus be expressed as $\hat{\sigma}_{\text {res }}^{\mathrm{TE}} \approx-\hat{\boldsymbol{\theta}}$ and $\hat{\sigma}_{\text {res }}^{\mathrm{TM}} \approx \hat{\boldsymbol{\rho}}$, where we have chosen to neglect the azimuthal polarisation component of the TM mode due to its small magnitude relative to the radial component. Note that we shall use the caret notation exclusively to denote unit vectors for which $|\hat{\mathbf{u}}|=1$. The interior electric field distribution $(\rho \leq P)$ can thus be expressed as

$$
\mathbf{E}_{\mathbf{m}}^{v}(\mathbf{r}) \approx \mathcal{E}_{0} \exp \left[-\frac{\theta^{2}}{2 \Theta_{m}^{2}}\right] H_{p}\left[\frac{\theta}{\Theta_{m}}\right] \operatorname{Ai}\left[f_{\mathbf{m}}^{v}(\rho)\right] e^{i m \phi} \hat{\sigma}_{\text {res }}^{v},
$$
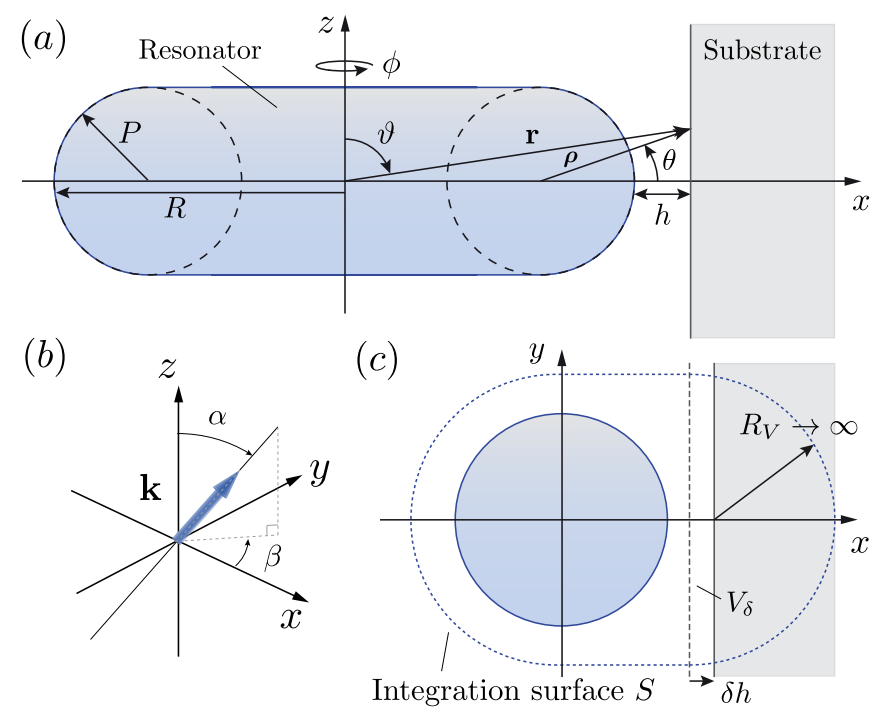

Fig. 1. WGM resonator geometry: (a) Definition of the coordinate system and geometrical parameters of an axisymmetric resonator perturbed by a planar substrate. (b) Coordinate system in the far field defining the angular dependence of a plane wave component with wavevector $\mathbf{k}$ (c) Schematic of integration volume and surface used to evaluate the resonance shift and mode broadening upon an infinitesimal shift of the dielectric substrate away from the resonator.

where, with reference to Figure $1(\mathrm{a}), \mathbf{r}=(x, y, z)$ is the position vector relative to the centre of the resonator, $H_{p}[z]$ are the Hermite polynomials of degree $p=0,1, \ldots, \mathbf{m}=(m, p, q)$ jointly describes the azimuthal, polar and radial indices, $v=$ TE or TM is the polarisation index and $\mathcal{E}_{0}$ is an arbitrary normalisation constant which is henceforth taken to be unity for simplicity. Note that highly localised WGMs correspond to $m \gg 1$ and small values of $p$ and $q(\sim 1)$. Eq. (1) was originally derived assuming a Dirchlet boundary condition at the resonator surface [43], however, we account for the openness of the resonator through use of the effective radius $\mathcal{R}_{v}=R+\Delta_{v}$ where $\Delta_{v}=\xi / \kappa$ describes the penetration depth of the WGM field into the host medium, $\kappa=k \sqrt{n^{2}-1}, \xi=1 / n^{2}=1 / n_{o}^{2}$ for TM modes and $\xi=1$, $n=n_{e}$ for TE modes, such that

$$
\begin{aligned}
f_{\mathbf{m}}^{v}(\rho) & =\left(P+\Delta_{v}-\rho\right) / u_{m}-\zeta_{q}, \\
\Theta_{m} & =\mathcal{R}_{v}^{3 / 4} P^{-3 / 4} m^{-1 / 2}, \\
u_{m} & =2^{-1 / 3} \mathcal{R}_{v} m^{-2 / 3},
\end{aligned}
$$

where $\zeta_{q}$ is the $q$-th root of the Airy function $\operatorname{Ai}[-\zeta]=0$. The validity and accuracy of use of the effective radius $\mathcal{R}_{v}$ has previously been verified [45, 46].

Due to the strongly confined nature of WGMs the total energy stored within the resonator volume, $V$, can be found by integrating over a toroidal volume defined by the local curvature of the resonator as shown in Figure 1, yielding

$$
U_{\mathbf{v}}^{\mathrm{res}} \approx \frac{1}{2} \epsilon_{0} n^{2} \int_{0}^{2 \pi} \int_{0}^{2 \pi} \int_{0}^{P}\left|\mathbf{E}_{\mathbf{m}}^{v}(\mathbf{r})\right|^{2} \rho|R-P+\rho \cos \theta| d \rho d \theta d \phi
$$

where we have also introduced the vector $\mathbf{v}=(\nu, m, p, q)$ containing all four mode indices. Throughout this work we shall 
freely switch between using the combined mode index $\mathbf{v}$ and separating the polarisation and scalar indices as $v$ and $\mathbf{m}$ depending on whether the polarisation index must be emphasised. For large $m$ and small $p$ the mode is highly localised near the outer surface $\rho \approx P$ and at the equator of the resonator whereby we can make the small angle approximation $\sin \theta \approx \theta \approx z / \rho$. Combining Eqs. (1) and (5) hence gives

$U_{\mathbf{v}}^{\mathrm{res}} \approx \pi \epsilon_{0} n^{2} R \int_{-\infty}^{\infty} \exp \left[-\frac{z^{2}}{P^{2} \Theta_{m}^{2}}\right] H_{p}^{2}\left[\frac{z}{P \Theta_{m}}\right] d z \int_{0}^{P} \operatorname{Ai}^{2}\left[f_{\mathbf{m}}^{v}(\rho)\right] d \rho$.

Use of the orthogonality relation of the Hermite functions $\int_{-\infty}^{\infty} \exp \left[-z^{2}\right] H_{p}[z] H_{a}[z] d z=\sqrt{\pi} 2^{p} p ! \delta_{a p}$ where $\delta_{a p}$ is the Kronecker delta, then yields

$$
U_{\mathbf{v}}^{\text {res }} \approx 2^{p} p ! \pi^{3 / 2} \epsilon_{0} u_{m} n^{2} \Theta_{m} R P\left[g_{\mathbf{v}}(0)-g_{\mathbf{v}}(P)\right]
$$

where $g_{\mathbf{v}}(\rho)=f_{\mathbf{m}}^{\nu}(\rho) \operatorname{Ai}\left[f_{\mathbf{m}}^{\nu}(\rho)\right]^{2}-\operatorname{Ai}^{\prime}\left[f_{\mathbf{m}}^{\nu}(\rho)\right]^{2}$ and the prime notation denotes differentiation with respect to the argument. For large $m$ we note that $g_{\mathbf{v}}(0) \approx 0$ and $g_{\mathbf{v}}(P) \approx-\mathrm{Ai}^{\prime}\left[-\zeta_{q}\right]^{2}$.

Exterior to the resonator $(\rho \geq P)$ the WGM exhibits an evanescent decay [46] viz.

$$
\mathbf{E}_{\mathbf{m}}^{v}(\mathbf{r}) \approx A_{v} \exp \left[-\frac{\theta^{2}}{2 \Theta_{m}^{2}}\right] H_{p}\left[\frac{\theta}{\Theta_{m}}\right] e^{-\kappa(\rho-P)} e^{i m \phi} \hat{\sigma}_{\text {sur }}^{v},
$$

where $A_{v}$ is a normalisation constant required to match the fields at the resonator surface, $\hat{\sigma}_{\text {sur }}^{\mathrm{TE}}=-\hat{\boldsymbol{\theta}}, \hat{\sigma}_{\text {sur }}^{\mathrm{TM}}=i a_{\text {sur }} \hat{\boldsymbol{\phi}}-b_{\text {sur }} \hat{\boldsymbol{\rho}}$ and $a_{\text {sur }} / b_{\text {sur }} \approx\left(1-n_{o}^{-2}\right)^{1 / 2}$ as follows from application of the Fresnel coefficients for light undergoing total internal reflection at a glancing angle to the resonator surface [47]. From applying the usual Maxwell boundary conditions it follows that $A_{\mathrm{TE}}=\mathrm{Ai}\left[\Delta_{\mathrm{TE}} / u_{m}-\zeta_{q}\right]$. Although within the bulk of the resonator the magnitude of the azimuthal component, which varies as $\sim \operatorname{Ai}^{\prime}\left[f_{\mathbf{m}}^{v}(\rho)\right]$, is negligible with respect to the radial field component (as per Eq. (1)), at the interior resonator surface it becomes $\left(n_{o}^{4}-n_{o}^{2}\right)^{1 / 2}$ times greater than that of the radial component. This ratio follows by recalling $a_{\text {sur }} / b_{\text {sur }} \approx\left(1-n_{o}^{-2}\right)^{1 / 2}$ and invoking the usual continuity conditions of the tangential and perpendicular electric field components. When considering the amplitude discontinuity of TM modes both field components must thus be included. With this in mind it can be shown that $A_{\mathrm{TM}}=\left(2 n_{o}^{4}-n_{o}^{2}\right)^{1 / 2} \mathrm{Ai}\left[\Delta_{\mathrm{TM}} / u_{m}-\zeta_{q}\right]$. Moreover, given Eq. (8) it follows that the relative contribution to the mode energy from the field outside the resonator is

$$
\frac{U_{\mathbf{v}}^{\text {sur }}}{U_{\mathbf{v}}^{\text {res }}} \approx \frac{A_{v}^{2} \int_{P}^{\infty} \exp [-2 \kappa(\rho-P)] d \rho}{n^{2} \int_{0}^{P} \operatorname{Ai}^{2}\left[f_{\mathbf{m}}^{v}(\rho)\right] d \rho} \approx \frac{A_{v}^{2}}{2 n^{2} \kappa u_{m} \mathrm{Ai}^{\prime}\left[-\zeta_{q}\right]^{2}}
$$

from which it quickly follows that $U_{\mathbf{v}}^{\text {res }} \gg U_{\mathbf{v}}^{\text {sur }}$ such that the majority of the mode energy is seen to lie within the resonator. Eq. (9) holds within the approximation that the field outside the resonator decays exponentially, however, similar expressions can also be derived through use of the complete mode profile $[5,48]$. Although expressions for the WGM mode profile and energy given in this section are approximate in nature, we have numerically verified the accuracy through comparison with rigorous finite element calculations performed using the method described in Ref. [49].

\section{MODE DISTRIBUTIONS IN THE PRESENCE OF AN ANISOTROPIC INTERFACE}

Interaction of a WGM with a dielectric perturbation, gives rise to a redistribution of the mode profile. This phenomenon has been well studied for the case of perturbing dielectric and plasmonic nanoparticles, in addition to consideration of layered structures $[33,35,50,51]$. Changes in the properties of morphological dependent resonances of a dielectric sphere near a conducting plane have also been considered using a multipolar expansion $[52,53]$. Here we consider the mode distribution, $\mathbf{E}_{\mathbf{v}}(\mathbf{r} ; h)$, resulting from interaction of a WGM with a semi-infinite dielectric substrate whose interface is placed at a distance $h$ from the surface of a $z$-cut birefringent resonator and with its normal directed along $\hat{\mathbf{x}}$ as depicted in Figure 1(a). The mode profiles defined in Eqs. (1) and (8) represent the limiting case $h \rightarrow \infty$. Moreover, we consider a uniaxial dielectric substrate with arbitrary optic axis $\hat{\mathbf{c}}$ and (extra-)ordinary refractive index $\left(n_{e}^{\text {sub }}\right) n_{o}^{\text {sub }}$. To account for potential back coupling into the resonator from reflection of the WGM from the interface, we first consider surface dressing of the WGM amplitude. With this in hand, we then proceed to derive the mode distribution in both the resonator interior and exterior, in addition to that in the infinite half space of the anisotropic dielectric, using an angular spectrum approach.

\section{A. Surface dressed scattering amplitudes}

To describe excitation of WGMs in an isolated resonator structure, we can consider an arbitrary incident field, which we represent as a superposition of modes viz.

$$
\mathbf{E}_{i}(\mathbf{r})=\sum_{\mathbf{v}} a_{\mathbf{v}} \mathbf{V}_{\mathbf{v}}(\mathbf{r})
$$

Assuming the modes form a complete orthogonal basis over the surface, $A$, of the resonator it follows that

$$
a_{\mathbf{v}}=\frac{\iint_{A} \mathbf{V}_{\mathbf{v}}^{*}(\mathbf{r}) \cdot \mathbf{E}_{i}(\mathbf{r}) d A}{\iint_{A} \mathbf{V}_{\mathbf{v}}^{*}(\mathbf{r}) \cdot \mathbf{V}_{\mathbf{v}}(\mathbf{r}) d A}
$$

The incident field gives rise to a scattered field, which can again be represented as a superposition of modes as

$$
\mathbf{E}_{S}(\mathbf{r} ; h \rightarrow \infty)=\sum_{\mathbf{v}} b_{\mathbf{v}} \mathbf{W}_{\mathbf{v}}(\mathbf{r}),
$$

where the additional parametric dependence on $h$ is introduced for later convenience. Note that we consider different modes $\mathbf{V}_{\mathbf{v}}$ and $\mathbf{W}_{\mathbf{v}}$ due to differing physical requirements at infinity. Specifically, the scattered modes must satisfy the Sommerfeld radiation condition, whereas the incident modes have a zero net energy flow through a closed surface and physically cannot possess a singularity in the volume of interest. Through application of the Maxwell boundary conditions at the surface of the resonator, the incident and scattered mode coefficients can be related through the matrix equation $\mathbf{b}=\mathbb{N a}$, where $\mathbb{N}$ is the scattering matrix and $\mathbf{a}=\left[a_{\mathbf{v}}\right]\left(\mathbf{b}=\left[b_{\mathbf{v}}\right]\right)$ is a vector formed by stacking all the incident (scattered) mode coefficients. For a spherical resonator, the modes $\mathbf{V}_{\mathbf{v}}$ and $\mathbf{W}_{\mathbf{v}}$ would correspond to the vector multipole modes with a radial dependence described by the spherical Bessel functions and Hankel functions (of the first kind) respectively. Accordingly, $\mathbb{N}$ would be a diagonal matrix with non-zero elements given by the usual Mie scattering amplitudes [54]. Modal properties of resonances in isolated optical resonators, such as the associated resonance frequencies and line widths, can then be determined through analysis of the poles of the scattering matrix $\mathbb{N}$ [55]. The field in the interior of the resonator can similarly be written

$$
\mathbf{E}_{r}(\mathbf{r} ; \infty)=\sum_{\mathbf{v}} f_{\mathbf{v}} \mathbf{U}_{\mathbf{v}}(\mathbf{r})
$$


where the internal mode coefficients can also be related to the illumination coefficients through $\mathbf{f}=\mathbb{Z} \mathbf{a}$.

Introduction of a dielectric inhomogeneity in the resonator surroundings, e.g. a dielectric interface or a perturbing nanoparticle, produces two additional contributions to the total field exterior to the resonator. Firstly, the incident field $\mathbf{E}_{i}$ is reflected from the inhomogeneity giving rise to a field $\mathbf{E}_{i r}$. Similarly, the field scattered from the resonator is reflected giving rise to a field $\mathbf{E}_{s r}$. Each contribution can also be decomposed according to ${ }^{1}$

$$
\mathbf{E}_{i r}(\mathbf{r} ; h)=\sum_{\mathbf{v}} c_{\mathbf{v}}^{\prime} \mathbf{V}_{\mathbf{v}}(\mathbf{r}) \text { and } \quad \mathbf{E}_{s r}(\mathbf{r} ; h)=\sum_{\mathbf{v}} d_{\mathbf{v}}^{\prime} \mathbf{V}_{\mathbf{v}}(\mathbf{r}) .
$$

The total field incident upon the resonator is that formed from the superposition of the incident and the reflected fields, i.e. $\mathbf{E}_{i}^{\text {eff }}=\mathbf{E}_{i}+\mathbf{E}_{i r}+\mathbf{E}_{s r}$, such that the scattered field is

$$
\mathbf{E}_{S}(\mathbf{r} ; h)=\sum_{\mathbf{v}} b_{\mathbf{v}}^{\prime} \mathbf{W}_{\mathbf{v}}(\mathbf{r})
$$

where $\mathbf{b}^{\prime}=\mathbb{N}\left(\mathbf{a}+\mathbf{c}^{\prime}+\mathbf{d}^{\prime}\right)$ and $\mathbf{b}^{\prime}, \mathbf{c}^{\prime}$ and $\mathbf{d}^{\prime}$ are perturbed coefficient vectors defined analogously to above. Noting further, that the reflected incident field originates from the incident field, we may write $\mathbf{c}^{\prime}=\mathbb{J} \mathbf{a}$ and similarly for the reflected scattered field we have $\mathbf{d}^{\prime}=\mathbb{K} \mathbf{b}^{\prime}$. Solving for the scattered coefficients $\mathbf{b}^{\prime}$ in terms of the illumination coefficients $\mathbf{a}$, thus yields

$$
\mathbf{b}^{\prime}=[\mathbb{I}-\mathbb{N} \mathbb{K}]^{-1} \mathbb{N}[\mathbb{I}+\mathbb{J}] \mathbf{a} \triangleq \mathbb{N}_{\text {eff }} \mathbf{a}
$$

where II is the identity matrix and $\mathbb{N}_{\text {eff }}$ is an effective, or dressed, scattering matrix. Through an analogous argument it also follows that

$$
\mathbf{f}^{\prime}=\mathbb{Z}\left(\mathbb{I}+\mathbb{J}+\mathbb{K N}_{\text {eff }}\right) \mathbf{a} \triangleq \mathbb{Z}_{\text {eff }} \mathbf{a} .
$$

Excitation of WGMs is typically achieved through evanescent coupling using a prism or waveguide structure [37], such that the excitation field is non-negligible over only a small extent of the resonator surface. Practically, dielectric tuning is also achieved using an independent dielectric substrate [31, 42], such that the excitation field does not contribute to the total field at the substrate interface. Accordingly we can safely neglect the contribution of $\mathbf{E}_{i r}$ for our purposes (i.e. we assume $\mathbb{J}=\mathbf{O}$, where $\mathrm{O}$ is the null matrix). We briefly note that the effective scattering coefficient derived above is equivalent to that which would be found by considering multiple reflections of the WGM from the dielectric substrate and summing over all reflected orders.

\section{B. Prism induced mode coupling}

Notably, evaluation of the effective scattering and transmission matrices described by Eq. (16) and Eq. (17) requires determination of the complex coupling coefficients, $K_{\mathbf{u}, \mathbf{v}}$, which comprise $\mathbb{K}$, which we consider in detail in this section. Our derivation consists of three steps: firstly we determine the angular spectrum of the WGM with mode index $\mathbf{v}=(v, m, p, q)$ at the dielectric interface; secondly, each constituent plane wave component is reflected from the interface by means of generalised Fresnel reflection coefficients; before finally, calculating the mode overlap between the reflected field with the WGM of order $\mathbf{u}=(\mu, l, a, b)=(\mu, \mathbf{l})$ (defined analogously to $\mathbf{v}$ ).

\footnotetext{
${ }^{1}$ Note that the choice of modes to use in this decomposition, in general, depends on the position $\mathbf{r}$ at which the field is considered. In this work we assume that the dielectric inhomogeneity is located exterior to the resonator and we consider the field close to the resonator such that Eq. (14) holds.
}

The first step of our derivation requires the angular spectrum $\widetilde{\mathbf{E}}_{\mathbf{m}}^{v}(\mathbf{k} ; h)$ of a given WGM at the dielectric interface located at $x_{0}=R+h$. Restricting our attention to large $m$ WGMs we can legitimately make a small angle approximation, and with reference to Figure $1(\mathrm{a})$, it follows that $\hat{r} \approx \hat{\rho}$ and $\hat{\boldsymbol{\theta}} \approx-\hat{\boldsymbol{\vartheta}}$. The symmetry of the TE and TM WGMs therefore implies the angular spectrum of the field is of the form $\widetilde{\mathbf{E}}_{\mathbf{m}}^{\mathrm{TE}}(\mathbf{k} ; h) \approx \widetilde{E}_{\mathbf{m}}^{\mathrm{TE}}(\mathbf{k} ; h) \hat{\boldsymbol{\alpha}}$ and $\widetilde{\mathbf{E}}_{\mathbf{m}}^{\mathrm{TM}}(\mathbf{k} ; h) \approx \widetilde{E}_{\mathbf{m}}^{\mathrm{TM}}(\mathbf{k} ; h) \hat{\boldsymbol{\beta}}$, where $\mathbf{k}=k[\sin \alpha \cos \beta, \sin \alpha \sin \beta, \cos \alpha]$, and, $\alpha$ and $\beta$ are the (complex) polar and azimuthal angles in $\mathbf{k}$ space (analogous to $\vartheta$ and $\phi)$ as depicted in Figure 1(b). We note that the radial polarisation component is absent in the far field so as to ensure transversality of the field. The scalar amplitudes, evaluated in a given $x$ plane, are given by

$$
\widetilde{E}_{\mathbf{m}}^{v}(\mathbf{k} ; x-R)=\frac{1}{4 \pi^{2}} \iint_{-\infty}^{\infty} E_{\mathbf{m}}^{v}(x, y, z) e^{-i\left(k_{y} y+k_{z} z\right)} d y d z
$$

where $E_{\mathbf{m}}^{v}(x, y, z)$ is the complex amplitude of $\mathbf{E}_{\mathbf{m}}^{v}(x, y, z)$ and we parameterise the amplitudes in terms of the distance $x-R$ of the $x$ plane from the resonator rim for later convenience. At the dielectric interface we have $x=x_{0}=(R-P+\rho \cos \theta) \cos \phi$, which for small $\theta$ and $\phi$ gives $\rho \approx P+h+(P+h) \theta^{2} / 2+(R+$ h) $\phi^{2} / 2$. Together with Eq. (8) we thus find

$$
\begin{aligned}
E_{\mathbf{m}}^{v}\left(x_{0}, y, z\right) & \approx A_{v} H_{p}\left[\frac{z}{P \Theta_{m}}\right] \exp \left[-\kappa h+i m \frac{y}{R}\right] \\
& \times \exp \left[-\frac{1}{2}\left\{\frac{y^{2}}{\Delta y^{2}}+\frac{z^{2}}{\Delta z^{2}}+\frac{z^{2}}{P^{2} \Theta_{m}^{2}}\right\}\right],
\end{aligned}
$$

where $\Delta y^{2}=R / \kappa, \Delta z^{2}=P / \kappa$ and we have also used the fact that $R \gg h$ and $P \gg h$. Eq. (19) represents the product of the WGM mode with a Gaussian coupling window, such that use of the convolution theorem yields

$$
\begin{aligned}
\widetilde{E}_{\mathbf{m}}^{v}(\mathbf{k} ; h) \approx & \widetilde{A}_{v} \exp \left[-\frac{1}{2}\left\{\left(k_{y}-k_{y r}\right)^{2} \Delta y^{2}+k_{z}^{2} \delta z^{2}\right\}\right] \exp [-\kappa h] \\
& \times\left[\sum_{q=0}^{p / 2} \frac{p !(-i)^{p-2 q}}{q !(p-2 q) !} \frac{(-2)^{q}}{\left(1+\sigma^{2}\right)^{q}} H_{p-2 q}\left[\frac{k_{z} \delta z^{2}}{P \Theta_{m}}\right]\right]
\end{aligned}
$$

where $\widetilde{A}_{v}=A_{v} \delta z \Delta y /(2 \pi), \delta z^{-2}=\Delta z^{-2}+\left(P \Theta_{m}\right)^{-2}$ and $\sigma=$ $\Delta z /\left(P \Theta_{m}\right)$. From Eq. (20) we see that as a consequence of the small angle approximation the angular spectrum is separable in $k_{y}$ and $k_{z}$. Moreover, in the $k_{y}$ direction the angular spectrum is centred on $k_{y r}=m / R$ (i.e. the propagation constant of the associated (WGM) and has a width of $1 / \Delta y \ll k$, whereas in the $k_{z}$ direction the spectrum is centred around $k_{z}=0$ and has a width $\sim 1 / \delta z \ll k$.

Reflection and transmission of each angular component can be described through use of generalised Fresnel reflection and transmission coefficients, a derivation of which can be found in Appendix A. We note, however, that the transmission coefficients act on the $\hat{\mathbf{s}}=(\mathbf{k} \times \hat{\mathbf{x}}) /|\mathbf{k} \times \hat{\mathbf{x}}|$ and $\hat{\mathbf{p}}=(\mathbf{k} \times \hat{\mathbf{s}}) /|\mathbf{k} \times \hat{\mathbf{s}}|$ field components, i.e. those perpendicular and parallel to the plane of incidence. It is therefore necessary to express the $\hat{\alpha}$ and $\hat{\beta}$ field components in terms of the $\hat{\mathbf{s}}$ and $\hat{\mathbf{p}}$ basis. Upon making the usual small angle approximation we find $\widetilde{\mathbf{E}}_{\mathbf{m}}^{\mathrm{TE}}(\mathbf{k} ; h) \approx \widetilde{E}_{\mathbf{m}}^{\mathrm{TE}}(\mathbf{k} ; h) \hat{\mathbf{s}}$ and $\widetilde{\mathbf{E}}_{\mathbf{m}}^{\mathrm{TM}}(\mathbf{k} ; h) \approx \widetilde{E}_{\mathbf{m}}^{\mathrm{TM}}(\mathbf{k} ; h) \hat{\mathbf{p}}$. The reflected angular spectrum in each case, denoted $\widetilde{\mathbf{E}}_{\mathbf{m}, r}^{v}\left(\mathbf{k}_{r} ; h\right)$, is hence

$$
\begin{aligned}
& \widetilde{\mathbf{E}}_{\mathbf{m}, r}^{\mathrm{TE}}\left(\mathbf{k}_{r} ; h\right)=\widetilde{E}_{\mathbf{m}}^{\mathrm{TE}}(\mathbf{k} ; h)\left[r_{s s} \hat{\mathbf{s}}_{r}+r_{s p} \hat{\mathbf{p}}_{r}\right] \\
& \widetilde{\mathbf{E}}_{\mathbf{m}, r}^{\mathrm{TM}}\left(\mathbf{k}_{r} ; h\right)=\widetilde{E}_{\mathbf{m}}^{\mathrm{TM}}(\mathbf{k} ; h)\left[r_{p s} \hat{\mathbf{s}}_{r}+r_{p p} \hat{\mathbf{p}}_{r}\right],
\end{aligned}
$$


where $\mathbf{k}_{r}=\left[-k_{x}, k_{y}, k_{z}\right]$ is the reflected wavevector, $\hat{\mathbf{s}}_{r}=\hat{\mathbf{s}}, \hat{\mathbf{p}}_{r}=$ $\left[\mathbf{k}_{r} \times \hat{\mathbf{s}}\right] /\left|\mathbf{k}_{r} \times \hat{\mathbf{s}}\right|$ and the reflection coefficients, $r_{i j}$, are defined in Appendix A. Accordingly the reflected field at a general position $\mathbf{r}$ exterior to the resonator is given by

$$
\begin{aligned}
& \mathbf{E}_{\mathbf{m}, r}^{\mathrm{TE}}(\mathbf{r} ; h)=\mathcal{E}_{\mathbf{m}}^{\mathrm{TE}, \mathrm{TE}}(\mathbf{r} ; h) \hat{\sigma}_{\mathrm{ref}}^{\mathrm{TE}}+\mathcal{E}_{\mathbf{m}}^{\mathrm{TE}, \mathrm{TM}}(\mathbf{r} ; h) \hat{\sigma}_{\mathrm{ref}}^{\mathrm{TM}} \\
& \mathbf{E}_{\mathbf{m}, r}^{\mathrm{TM}}(\mathbf{r} ; h)=\mathcal{E}_{\mathbf{m}}^{\mathrm{TM}, \mathrm{TE}}(\mathbf{r} ; h) \hat{\sigma}_{\mathrm{ref}}^{\mathrm{TE}}+\mathcal{E}_{\mathbf{m}}^{\mathrm{TM}, \mathrm{TM}}(\mathbf{r} ; h) \hat{\sigma}_{\mathrm{ref}}^{\mathrm{TM}}
\end{aligned}
$$

as follows from reciprocity and where $\hat{\sigma}_{\text {ref }}^{\mathrm{TE}}=-\hat{\boldsymbol{\theta}}_{r}, \hat{\sigma}_{\mathrm{ref}}^{\mathrm{TM}}=$ $\left[i a_{\text {sur }} \hat{\boldsymbol{\phi}}_{r}-b_{\text {sur }} \hat{\boldsymbol{\rho}}_{r}\right], \hat{\boldsymbol{\rho}}_{r}=\hat{\boldsymbol{\rho}}(\theta, \pi-\phi)$ and similarly for $\hat{\boldsymbol{\theta}}_{r}$ and $\hat{\boldsymbol{\phi}}_{r}$. The amplitude factors are given by

$$
\begin{aligned}
\mathcal{E}_{\mathbf{m}}^{\mathrm{TE}, \mathrm{TE}}(\mathbf{r} ; h) & =\iint_{-\infty}^{\infty} r_{s s} \widetilde{E}_{\mathbf{m}}^{\mathrm{TE}}(\mathbf{k} ; h) \exp \left[i \mathbf{k}_{r} \cdot \Delta \mathbf{r}\right] d k_{y} d k_{z} \\
\mathcal{E}_{\mathbf{m}}^{\mathrm{TE}, \mathrm{TM}}(\mathbf{r} ; h) & =\iint_{-\infty}^{\infty} r_{s p} \widetilde{E}_{\mathbf{m}}^{\mathrm{TE}}(\mathbf{k} ; h) \exp \left[i \mathbf{k}_{r} \cdot \Delta \mathbf{r}\right] d k_{y} d k_{z} \\
\mathcal{E}_{\mathbf{m}}^{\mathrm{TM}, \mathrm{TE}}(\mathbf{r} ; h) & =\iint_{-\infty}^{\infty} r_{p s} \widetilde{E}_{\mathbf{m}}^{\mathrm{TM}}(\mathbf{k} ; h) \exp \left[i \mathbf{k}_{r} \cdot \Delta \mathbf{r}\right] d k_{y} d k_{z} \\
\mathcal{E}_{\mathbf{m}}^{\mathrm{TM}, \mathrm{TM}}(\mathbf{r} ; h) & =\iint_{-\infty}^{\infty} r_{p p} \widetilde{E}_{\mathbf{m}}^{\mathrm{TM}}(\mathbf{k} ; h) \exp \left[i \mathbf{k}_{r} \cdot \Delta \mathbf{r}\right] d k_{y} d k_{z} .
\end{aligned}
$$

where $\Delta \mathbf{r}=\left(x-x_{0}, y, z\right)=\mathbf{r}-x_{0} \hat{\mathbf{x}}$ is the position vector relative to the plane of the prism interface. Determination of the reflected field over the resonator surface requires evaluation of these integrals for $\mathbf{r} \in A$. To do so we make a number of further approximations. We once more note that $k_{y} \approx m / R$, $k_{z} \approx 0, \Delta k_{y} \ll k$ and $\Delta k_{z} \ll k$, such that $-i k_{x} x \approx \kappa x$. Furthermore given the small range of $k_{y}$ and $k_{z}$, we assert that the variation of the reflection coefficients is small, such that $r_{i j}\left(k_{y}, k_{z}\right) \approx r_{i j}(m / R, 0) \triangleq \bar{r}_{i j}$ for $\{i, j\}=\{s, p\}$ whereby the reflection coefficient can be factored outside of the integral. With these approximations Eqs. (25)-(28) essentially reduce to the 2D inverse Fourier transform of the angular spectra $\widetilde{E}_{\mathbf{m}}^{v}(\mathbf{k} ; h)$ with some additional Gaussian factors due to the additional evanescent decay of the mode. We thus find on the resonator surface, i.e. $(x, y, z) \in A$, that

$$
\begin{aligned}
\mathcal{E}_{\mathbf{m}}^{v, \mu}(x, y, z ; h) \approx A_{v} \bar{r}_{i j} H_{p}\left[\frac{z}{P \Theta_{m}}\right] \exp \left[-2 \kappa h+i m \frac{y}{R}\right] \\
\times \exp \left[-\left\{\frac{y^{2}}{\Delta y^{2}}+\frac{z^{2}}{\Delta z^{2}}+\frac{z^{2}}{2 P^{2} \Theta_{m}^{2}}\right\}\right],
\end{aligned}
$$

where $i=s(p)$ for $v=\mathrm{TE}(\mathrm{TM})$ and similarly for $j$. With this association understood we henceforth adopt the shorthand notation $\bar{r}_{v \mu}=\bar{r}_{i j}$. The coupling constant can then be evaluated viz. (c.f. Eq. (11))

$$
K_{\mathbf{u}, \mathbf{v}}=\frac{\iint_{A} \mathbf{E}_{\mathbf{l}}^{\mu *}(\mathbf{r}) \cdot \mathbf{E}_{\mathbf{m}, r}^{v}(\mathbf{r} ; h)}{\iint_{A} \mathbf{E}_{\mathbf{1}}^{\mu *}(\mathbf{r}) \cdot \mathbf{E}_{\mathbf{l}}^{\mu}(\mathbf{r})} d A \triangleq \frac{I_{\mathbf{u}, \mathbf{v}}^{(1)}}{I_{\mathbf{u}}^{(0)}}
$$

We first evaluate the integral $I_{\mathbf{u}}^{(0)}$ which follows easily from the orthogonality of the Hermite functions as

$$
I_{\mathbf{u}}^{(0)} \approx 2^{a+1} \pi^{3 / 2} A_{\mu}^{2} a ! R P \Theta_{l} .
$$

Turning attention to evaluation of $I_{\mathbf{u}, \mathbf{v}}^{(1)}$, we first consider the polarisation dependence. Within our small angle approximation it can be shown that $\hat{\sigma}_{\text {sur }}^{\nu, *} \cdot \hat{\sigma}_{\text {ref }}^{\mu} \approx 1$ for $v=\mu=\mathrm{TE}$ and $\approx k^{2} /\left(2 k_{y r}^{2}-k^{2}\right) \approx\left(2 n_{o}^{2}-1\right)^{-1}$ for $v=\mu=$ TM and zero otherwise. Moreover, the narrow windowing function described by the $\exp \left[-y^{2} / \Delta y^{2}\right]$ factor means that we need only perform the integration over a small region of the resonator surface whereby $d A \approx d y d z$. Use of Eqs. (19) and (29) then allows us to express $I_{\mathbf{u}, \mathbf{v}}^{(1)}$ in the form

$$
\begin{aligned}
I_{\mathbf{u}, \mathbf{v}}^{(1)} \approx \pi^{1 / 2} A_{v} A_{\mu} \bar{r}_{v \mu} \Delta y P \Theta_{m} I_{a p}^{(2)} \hat{\sigma}_{\text {sur }}^{\mu, *} \cdot \hat{\sigma}_{\text {ref }}^{\mu} \\
\times \exp [-2 \kappa h] \exp \left[-\frac{(m-l)^{2}}{2 \Delta m^{2}}\right]
\end{aligned}
$$

where $\Delta m^{2} \approx 2 m^{-1} \sqrt{1-n^{-2}}$. For large $m$ we have $\Delta m \ll$ 1 , such that the second exponential in Eq. (32) can be safely replaced by the Kronecker delta $\delta_{l, m}$ whereby $\Theta_{l}=\Theta_{m}$. The remaining integral term, $I_{a p}^{(2)}$, in Eq. (32) can then be written as

$$
I_{a p}^{(2)}=\int_{-\infty}^{\infty} H_{a}[w] H_{p}[w] \exp \left[-2 \tau^{2} w^{2}\right] d w
$$

with $\sqrt{2} \tau=P \Theta_{m} / \delta z$. It is evident that $I_{a p}^{(2)}$ is identically zero when $a$ and $p$ are of opposite parity, i.e. polar modes of differing symmetry do not couple as would be expected. When $a+p$ is even, however, $I_{a p}^{(2)}$ can be evaluated analytically [56] yielding

$$
\begin{array}{r}
I_{a p}^{(2)}=2^{\frac{a+p-1}{2}} s^{-a-p-1}\left(1-2 \tau^{2}\right)^{\frac{a+p}{2}} \Gamma\left[\frac{a+p+1}{2}\right] \\
\quad \times{ }_{2} F_{1}\left[-a,-p ; \frac{1-a-p}{2} ; \frac{\tau^{2}}{2 \tau^{2}-1}\right],
\end{array}
$$

where $\Gamma[n]$ and ${ }_{2} F_{1}[a, b ; c ; z]$ are the Gamma and Gauss hypergeometric functions respectively. Hence, we ultimately arrive at the desired coupling coefficients

$$
K_{\mathbf{u}, \mathbf{v}}=\delta_{l, m} \frac{\bar{r}_{v \mu} \Delta y I_{a p}^{(2)}}{2^{a+1} \pi a ! R} e^{-2 \kappa h} \times \begin{cases}1 & \mu=\mathrm{TE} \\ \left(2 n_{o}^{2}-1\right)^{-1} & \mu=\mathrm{TM} .\end{cases}
$$

A heat map depicting the interface induced coupling strength between modes of different polar orders ( $a$ and $p$ ) as described by Eq. (35) is shown in Figure 2. Note that for ease of comparison we have also included a further normalisation factor of $2^{p} p$ ! in the data shown in Figure 2, which arises from the scaling of the initial mode energy (Eq. (5)). Inspection of Eq. (35) and Figure 2 reveals that a WGM of order $p$ couples most strongly to the lowest order WGM of the same symmetry upon reflection, i.e. the fundamental $a=0$ mode for even $p$ or the $a=1$ mode for odd $p$. This is a direct consequence of the finite width coupling window. We reiterate that coupling between modes of differing symmetry is not possible. Moreover, as follows from the conservation of angular momentum, coupling between modes with different azimuthal indices ( $l$ and $m$ ) is also forbidden. Eq. (35), however, does not forbid coupling between modes of differing polarisation, albeit, within our small angle approximation, such polarisation mixing stems from the anisotropy of the substrate and would be absent for an isotropic prism.

\section{Mode distributions}

Having described the reflection of a WGM by a dielectric substrate, we now determine the complete perturbed mode distributions. WGMs with differing mode indices are typically spectrally distinct in most resonators (with the exception of a perfectly spherical resonator for which the polar modes are degenerate) as is reflected in the amplitude of the scattering coefficients, $\eta_{\mathbf{v}}$ and $\zeta_{\mathbf{v}}$, contained in $\mathbb{N}$ and $\mathbb{Z}$ respectively. Consequently, assuming $R \neq P$, we can make a single mode approximation whereby Eq. (16), simplifies to

$$
b_{\mathbf{v}}^{\prime} \approx \frac{\eta_{\mathbf{v}}}{1-\eta_{\mathbf{v}} K_{\mathbf{v}, \mathbf{v}}} a_{\mathbf{v}} \triangleq \eta_{\mathbf{v}, \text { eff }} a_{\mathbf{v}} .
$$




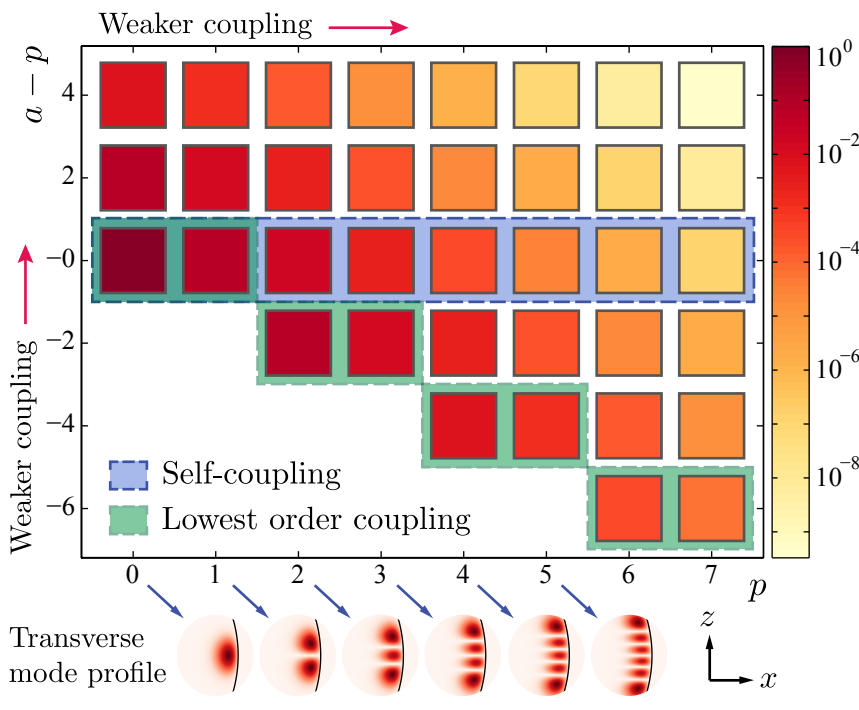

Fig. 2. Interface induced coupling strengths: Heat map of the coupling coefficients between a WGM of polar order $p$ to a WGM of polar order $a$, as given by $\left|I_{a p}^{(2)}\right| /\left[2^{a+p+1} p ! a !\right]$. A value of $\tau=1.2$ was assumed. Mode profiles of differing polar orders are shown in the lower inset from which different oddeven symmetry classes are apparent. Strongest coupling of a given WGM order $p$ is to the lowest order mode of the same symmetry as highlighted by the green boxes, however, coupling to modes of a different symmetry class is not possible.

Close to resonance and initially neglecting possible material absorption in the resonator, the scattering coefficient can be approximated by the Breit-Wigner line shape [57] viz.

$$
\eta_{\mathbf{v}}(\omega)=-\frac{\gamma_{\mathbf{v}, \text { rad }} / 2}{\gamma_{\mathbf{v}, \text { rad }} / 2-i\left(\omega-\omega_{\mathbf{v}}\right)}
$$

where $\omega_{\mathbf{v}}$ and $\gamma_{\mathbf{v} \text {,rad }}$ denote the resonance frequency and radiative line width respectively. The -1 pre-factor follows from imposing field continuity at the resonator surface. As shown in Appendix B material absorption in the resonator reduces the magnitude of the peak scattering amplitude such that

$$
\eta_{\mathbf{v}}(\omega)=-\frac{\gamma_{\mathbf{v}, \text { rad }} / 2}{\left(\gamma_{\mathbf{v}, \text { rad }}+\gamma_{\mathbf{v}, \text { abs }}\right) / 2-i\left(\omega-\omega_{\mathbf{v}}\right)}
$$

where $\gamma_{\mathbf{v}, \text { abs }}$ is the absorptive line width [58]. Similarly Eq. (17) simplifies to

$$
f_{\mathbf{v}}^{\prime} \approx \frac{\zeta_{\mathbf{v}}}{1-\eta_{\mathbf{v}} K_{\mathbf{v}, \mathbf{v}}} a_{\mathbf{v}} \triangleq \zeta_{\mathbf{v}, \text { eff }} a_{\mathbf{v}}
$$

Combination of Eqs. (36), (38) and (39) shows that on resonance the scattered and internal mode coefficients $b_{\mathbf{v}}^{\prime}$ and $f_{\mathbf{v}}^{\prime}$ are reduced by a factor of $\mathcal{A}(h)=\left(1-\eta_{\mathbf{v}} K_{\mathbf{v}, \mathbf{v}}\right)^{-1} \approx\left(\gamma_{\mathbf{v}, \text { rad }}+\right.$ $\left.\gamma_{\mathbf{v}, \text { abs }}\right) /\left(\gamma_{\mathbf{v}, \text { rad }}+\gamma_{\mathbf{v} \text {,abs }}+\gamma_{\mathbf{v} \text {,rad }} K_{\mathbf{v}, \mathbf{v}}\right)$ relative to the unperturbed $(h \rightarrow \infty)$ case. For absorption limited resonators for which $\gamma_{\mathbf{v} \text {,abs }} \gg \gamma_{\mathbf{v} \text {,rad }}$ we find $\mathcal{A}(h) \approx 1$ and hence $b_{\mathbf{v}}^{\prime} \approx b_{\mathbf{v}}$ and $f_{\mathbf{v}}^{\prime} \approx f_{\mathbf{v}}$. Within the resonant mode approximation, it therefore immediately follows that the WGM field distribution within the resonator $(\rho<P)$ in the presence of a dielectric interface is given simply by $\mathbf{E}_{\mathbf{v}}(\mathbf{r} ; h)=\mathcal{A}(h) \mathbf{E}_{\mathbf{v}}(\mathbf{r} ; \infty)$, where $\mathbf{E}_{\mathbf{v}}(\mathbf{r} ; \infty)$ is given by Eq. (1). Recalling results from above we also find the field exterior to the resonator $\left(\rho \geq P, x<x_{0}\right)$ is given by $\mathbf{E}_{\mathbf{v}}(\mathbf{r} ; h)=\mathcal{A}(h)\left[\mathbf{E}_{\mathbf{v}}(\mathbf{r} ; \infty)+\mathbf{E}_{\mathbf{v}, r}(\mathbf{r} ; h)\right]$, where now $\mathbf{E}_{\mathbf{v}}(\mathbf{r} ; \infty)$ is given by Eq. (8) and $\mathbf{E}_{\mathbf{v}, r}(\mathbf{r} ; h)$ follows from Eqs. (23)-(28). Following the same line of arguments used to derive Eq. (29) we can, however, write

$$
\mathbf{E}_{\mathbf{m}, r}^{v}(\mathbf{r} ; h) \approx \sum_{\mu \in\{\mathrm{TE}, \mathrm{TM}\}} \bar{r}_{\nu \mu} \exp [-2 \kappa h] E_{\mathbf{v}}(-x, y, z ; \infty) \hat{\sigma}_{\text {ref }}^{\mu} .
$$

The field transmitted into the volume of the anisotropic substrate $x \geq x_{0}$ can be found similarly to the reflected field using the generalised Fresnel transmission coefficients (Appendix A). From the preceding analysis in Section $3 \mathrm{~B}$ we note that for an initially unperturbed WGM of order $\mathbf{v}$, the component of the perturbed field incident on the prism interface is given by $\mathcal{A}(h) \mathbf{E}_{\mathbf{m}}^{v}\left(x_{0}, y, z ; \infty\right)$, with a corresponding angular spectrum $\mathcal{A}(h) \widetilde{\mathbf{E}}_{\mathbf{m}}^{v}(\mathbf{k} ; h)$. Upon transmission, each constituent plane wave generates an ordinary and extraordinary wave in the prism, with associated wavevectors $\mathbf{k}_{o}$ and $\mathbf{k}_{e}$, such that the transmitted field takes the form

$$
\mathbf{E}_{\mathbf{m}}^{v}(\mathbf{r} ; h)=\mathcal{A}(h) \sum_{j \in\{o, e\}} \iint_{-\infty}^{\infty} t_{i j} \widetilde{E}_{\mathbf{m}}^{v}(\mathbf{k} ; h) \hat{\mathbf{j}} e^{i \mathbf{k}_{j} \cdot \Delta \mathbf{r}} d k_{y} d k_{z},
$$

where the subscript $i=s, p$ for $v=\mathrm{TE}$, TM respectively, $t_{i j}(j \in$ $\{o, e\})$ are the generalised Fresnel transmission coefficients and $\hat{\mathbf{j}}=\hat{\mathbf{o}}$ and $\hat{\mathbf{e}}$ are the unit polarisation vectors for the ordinary and extraordinary waves respectively. The explicit dependence of $t_{i j}$, $\mathbf{k}_{o}$ and $\mathbf{k}_{e}$ on the incident wavevector $\mathbf{k}$ is given in Appendix A. To simplify Eq. (41) further we note that exponential terms vary rapidly with $k_{y}$ and $k_{z}$. In comparison the Fresnel coefficients and polarisation terms vary weakly within the limited range of $k_{y}$ and $k_{z}$ over which $\widetilde{\mathbf{E}}_{\mathbf{m}}^{v}$ is non-negligible. As such we make the approximations $\hat{\mathbf{o}} \approx\left(\overline{\mathbf{k}}_{o} \times \hat{\mathbf{c}}\right) /\left|\overline{\mathbf{k}}_{o} \times \hat{\mathbf{c}}\right|$ and $\hat{\mathbf{e}} \approx \stackrel{\leftrightarrow}{\epsilon}-1\left(\overline{\mathbf{k}}_{e} \times\right.$ $\left.\left[\overline{\mathbf{k}}_{e} \times \hat{\mathbf{c}}\right]\right) /\left|\stackrel{\leftrightarrow}{\epsilon}-1\left(\overline{\mathbf{k}}_{e} \times\left[\overline{\mathbf{k}}_{e} \times \hat{\mathbf{c}}\right]\right)\right|$, where $\overline{\mathbf{k}}_{o}$ and $\overline{\mathbf{k}}_{e}$ are the central ordinary and extraordinary wavevectors found from Eqs. (77)(81) with $k_{y}=k_{y r}$ and $k_{z}=0$. We hence obtain

$$
\mathbf{E}_{\mathbf{m}}^{v}(\mathbf{r} ; h)=\mathcal{A}(h) \sum_{j \in\{0, e\}} \bar{t}_{i j} \hat{\mathbf{j}} \iint_{-\infty}^{\infty} \widetilde{E}_{\mathbf{m}}^{v}(\mathbf{k} ; h) e^{i k_{j, x} \Delta x} e^{i\left(k_{y} y+k_{z} z\right)} d k_{y} d k_{z}
$$

where $\Delta x=x-x_{0}$ and $\bar{t}_{i j}$ is defined analogously to $\bar{r}_{i j}$ above. Once more taking advantage of the small angular spread in $k_{y}$ and $k_{z}$ we expand the $k_{j, x}$ exponent around $k_{y}=k_{y r}$ and $k_{z}=0$ which to leading order yields

$$
\begin{aligned}
& k_{o, x} \approx\left(n_{o}^{2} k^{2}-k_{y} k_{y r}\right) /\left(n_{o}^{2} k^{2}-k_{y r}^{2}\right)^{1 / 2} \triangleq \chi_{o}^{(0)}+\chi_{o}^{(1)} k_{y} \\
& k_{e, x} \approx \frac{2 k^{2}}{\bar{D}^{1 / 2}}+\frac{k_{y}}{k_{y r}}\left[\frac{\bar{v}+\bar{D}^{1 / 2}}{2 u}-\frac{2 k^{2}}{\bar{D}^{1 / 2}}\right] \triangleq \chi_{e}^{(0)}+\chi_{e}^{(1)} k_{y}
\end{aligned}
$$

where $\bar{D}=\bar{v}^{2}-4 u \bar{w}, \bar{v}=v\left(k_{y r}, 0\right)$ and $\bar{w}=w\left(k_{y r}, 0\right)$ follow from Eqs. (79)-(81) in Appendix A. The constant terms in Eqs. (43) and (44) can be factored out of the integrals of Eq. (42) such that the integrals are now of the form of a simple Fourier transform with respect to the variables $k_{y}$ and $k_{z}$. Accordingly, the terms linear in $k_{y}$ can be simply accounted for by recalling the shift theorem, whereby Eq. (42) becomes

$$
\mathbf{E}_{\mathbf{m}}^{v}(\mathbf{r} ; h)=\mathcal{A}(h) \sum_{j \in\{0, e\}} \bar{t}_{i j} \hat{\mathbf{j}} e^{i \chi_{j}^{(0)} \Delta x} E_{\mathbf{m}}^{v}\left(x_{0}, y+\chi_{j}^{(1)} \Delta x, z ; \infty\right)
$$


where $\chi_{j}^{(0)}$ and $\chi_{j}^{(1)}$ are defined by Eqs. (43) and (44). We note that near the critical angle, the variation of the transmission coefficients $t_{i j}$ can vary strongly with $k_{y}$ such that the approximations leading to Eq. (45) are not valid. In this case the full integral expressions of Eq. (41) must be used to account for Fresnel filtering effects $[59,60]$. Similar restrictions also apply to calculation of the reflected field and mode coupling as discussed in Section 3B.

\section{PRISM INDUCED RESONANCE PERTURBATIONS}

Shifts in the resonance frequency of modes in a closed cavity induced by local dielectric perturbations can be described by the Bethe-Schwinger equation [61]. Moreover, it has recently been shown (and experimentally verified) that this formula can also be used to account for mode broadening and radiative shifts in open resonators if the far-field components are incorporated [44]. In Appendix $C$ we briefly present a derivation of the Bethe-Schwinger equation for open cavities in the presence of anisotropic dielectric perturbations, which reads

$$
\begin{aligned}
& -i \oint_{S}\left(\delta \mathbf{E}_{\mathbf{v}} \times \mathbf{H}_{\mathbf{v}}^{*}+\mathbf{E}_{\mathbf{v}}^{*} \times \delta \mathbf{H}_{\mathbf{v}}\right) \cdot d \mathbf{S}=\int_{V}\left[\bar{\omega}_{\mathbf{v}}^{\prime}\left(\mathbf{E}_{\mathbf{v}}^{*} \cdot \mathbf{D}_{\mathbf{v}}^{\prime}+\mathbf{H}_{\mathbf{v}}^{*} \cdot \mathbf{B}_{\mathbf{v}}^{\prime}\right)\right. \\
& \left.-\bar{\omega}_{\mathbf{v}}\left(\mathbf{E}_{\mathbf{v}}^{*} \cdot \mathbf{D}_{\mathbf{v}}+\mathbf{H}_{\mathbf{v}}^{*} \cdot \mathbf{B}_{\mathbf{v}}\right)-\bar{\omega}_{\mathbf{v}}^{*}\left(\delta \mathbf{E}_{\mathbf{v}} \cdot \mathbf{D}_{\mathbf{v}}^{*}+\delta \mathbf{H}_{\mathbf{v}} \cdot \mathbf{B}_{\mathbf{v}}^{*}\right)\right] d V
\end{aligned}
$$

where $\bar{\omega}_{\mathbf{v}}=\omega_{\mathbf{v}}-i \gamma_{\mathbf{v}} / 2$ is the complex resonance frequency, $\delta \mathbf{E}_{\mathbf{v}}=\mathbf{E}_{\mathbf{v}}^{\prime}-\mathbf{E}_{\mathbf{v}}, \mathbf{E}_{\mathbf{v}}$ and $\mathbf{E}_{\mathbf{v}}^{\prime}$ are unperturbed and perturbed electric field distributions respectively (similarly for the magnetic field $\mathbf{H}_{\mathbf{v}}$ ), $\mathbf{D}_{\mathbf{v}}=\overleftrightarrow{\epsilon} \mathbf{E}_{\mathbf{v}}, \mathbf{B}_{\mathbf{v}}=\overleftrightarrow{\mu} \mathbf{H}_{\mathbf{v}}$, and $\overleftrightarrow{\epsilon}$ and $\overleftrightarrow{\mu}$ are the spatially dependant electric permittivity and magnetic permeability tensors. The volume $V$ and the associated surface $S$ over which the integrals Eq. (46) are taken are shown in Figure 1(c) (see also Appendix C). Although ultimately it is our goal to determine the total resonance shift and line width broadening induced by the presence of a uniaxial substrate relative to the case in which no substrate is present, the perturbed and unperturbed modes required to evaluate the Bethe-Schwinger equation can not be taken as $\mathbf{E}_{\mathbf{v}}(\mathbf{r} ; \infty)$ and $\mathbf{E}_{\mathbf{v}}(\mathbf{r} ; h)$ given above. This can intuitively be seen, since the second term in Eq. (46) can be associated with radiative losses in the far field, however, this term evaluates to zero if the unperturbed mode $\mathbf{E}_{\mathbf{v}}(\mathbf{r} ; \infty)$ is chosen because in the far field $\left|\mathbf{E}_{\mathbf{v}}(\mathbf{r} ; \infty)\right| \rightarrow 0$. Physically such a scenario is incorrect since radiative losses give rise to a mode broadening which is not accounted for. Instead, we consider the change of the WGM frequency and lifetime assuming that the dielectric is initially located at a distance $h$ from the resonator and is then displaced by an infinitesimally small distance, $\delta h$ away from the resonator. Accordingly, after making the further assumptions described in Appendix C,the Bethe-Schwinger equation takes the form

$$
\delta \bar{\omega}_{\mathbf{v}}=\bar{\omega}_{\mathbf{v}}(h+\delta h)-\bar{\omega}_{\mathbf{v}}(h)=-\left[\bar{\omega} N_{\mathbf{v}}^{(1)}+i N_{\mathbf{v}}^{(2)}\right] / N_{\mathbf{v}}^{(0)},
$$

where we have defined the integral terms (assuming $\delta \mu=\mathbb{O}$ )

$$
\begin{aligned}
& N_{\mathbf{v}}^{(0)}=\int_{V}\left\{\mathbf{E}_{\mathbf{v}}^{*}(\mathbf{r} ; h) \overleftrightarrow{\epsilon}\right. \mathbf{E}_{\mathbf{v}}(\mathbf{r} ; h+\delta h) \\
&\left.+\mathbf{H}_{\mathbf{v}}^{*}(\mathbf{r} ; h) \overleftrightarrow{\mu} \mathbf{H}_{\mathbf{v}}(\mathbf{r} ; h+\delta h)\right\} d V, \\
& N_{\mathbf{v}}^{(1)}=\int_{V_{\delta}} \mathbf{E}_{\mathbf{v}}^{*}(\mathbf{r} ; h) \delta \overleftrightarrow{\epsilon} \mathbf{E}_{\mathbf{v}}(\mathbf{r} ; h+\delta h) d V
\end{aligned}
$$

$$
\begin{aligned}
N_{\mathbf{v}}^{(2)}=\oint_{S}\left\{\delta \mathbf{E}_{\mathbf{v}}^{*}(\mathbf{r}) \times\right. & \mathbf{H}_{\mathbf{v}}(\mathbf{r} ; h+\delta h) \\
& \left.+\mathbf{E}_{\mathbf{v}}^{*}(\mathbf{r} ; h+\delta h) \times \delta \mathbf{H}_{\mathbf{v}}(\mathbf{r})\right\} \cdot d \mathbf{S} .
\end{aligned}
$$

and $\delta \mathbf{E}_{\mathbf{v}}(\mathbf{r})=\mathbf{E}_{\mathbf{v}}(\mathbf{r} ; h+\delta h)-\mathbf{E}_{\mathbf{v}}(\mathbf{r} ; h)$ (and similarly for $\left.\delta \mathbf{H}\right) . V_{\delta}$ defines the volume $h \leq x-R \leq h+\delta h$ for which $\delta \overleftrightarrow{\epsilon}=\epsilon_{0} \mathbb{I}-$ $\overleftrightarrow{\epsilon}^{\text {sub }}$ is non zero. The total change in the complex resonance frequency induced by the presence of the substrate can then be found by noting

$$
\frac{d \bar{\omega}}{d h}=\lim _{\delta h \rightarrow 0} \frac{\delta \bar{\omega}}{\delta h}
$$

such that

$$
\Delta \bar{\omega}_{\mathbf{v}}=\bar{\omega}_{\mathbf{v}}(h)-\bar{\omega}_{\mathbf{v}}(\infty)=\int_{\infty}^{h} \frac{d \bar{\omega}}{d h^{\prime}} d h^{\prime} .
$$

Each of the $N_{\mathbf{v}}$ terms defined in Eqs. (48)-(50) will now be considered in turn.

Evaluation of $N_{\mathbf{v}}^{(0)}$ : We initially consider evaluation of the volume integral $N_{\mathbf{v}}^{(0)}$. Since we consider an infinitesimal shift of the dielectric substrate it follows that the volume of the perturbation in $\overleftrightarrow{\epsilon}(\mathbf{r})$ is much smaller than the total volume considered. Accordingly we can approximate $N_{\mathbf{v}}^{(0)}$ as [44]

$$
N_{\mathbf{v}}^{(0)}=\int_{V}\left\{\mathbf{E}_{\mathbf{v}}^{*}(\mathbf{r} ; h) \overleftrightarrow{\epsilon} \mathbf{E}_{\mathbf{v}}(\mathbf{r} ; h)+\mathbf{H}_{\mathbf{v}}^{*}(\mathbf{r} ; h) \overleftrightarrow{\mu} \mathbf{H}_{\mathbf{v}}(\mathbf{r} ; h)\right\} d V
$$

which is four times the total energy of the WGM in the presence of a dielectric substrate placed at a distance $h$ from the resonator surface. We further approximate the latter, by the surface dressed mode energy of an unperturbed WGM, whereby

$$
N_{\mathbf{v}}^{(0)}=4|\mathcal{A}(h)|^{2} U_{\mathbf{v}}^{\text {res }}
$$

where $U_{\mathbf{v}}^{\text {res }}$ is the mode energy defined by Eqs. (5) and (7).

Evaluation of $N_{\mathbf{v}}^{(1)}$ : To evaluate $N_{\mathbf{v}}^{(1)}$ we note that within the domain of integration, $V_{\delta}$, the mode distribution before the interface is displaced is that which is transmitted into the substrate, i.e. that defined by Eq. (41). After the interface is shifted the field distribution is given by $\mathcal{A}(h+\delta h)\left[\mathbf{E}_{\mathbf{v}}(\mathbf{r})+\mathbf{E}_{\mathbf{v}, r}(\mathbf{r} ; h+\delta h)\right]$. From Eqs. (23) and (41) it therefore follows that

$$
\begin{aligned}
& N_{\mathbf{v}}^{(1)}=\mathcal{A}^{*}(h) \mathcal{A}(h+\delta h) \int_{V_{\delta}} \iint_{-\infty}^{\infty} \iint_{-\infty}^{\infty} \widetilde{E}_{\mathbf{v}}^{*}(\mathbf{k} ; h) \widetilde{E}_{\mathbf{v}}\left(\mathbf{k}^{\prime} ; h+\delta h\right)
\end{aligned}
$$

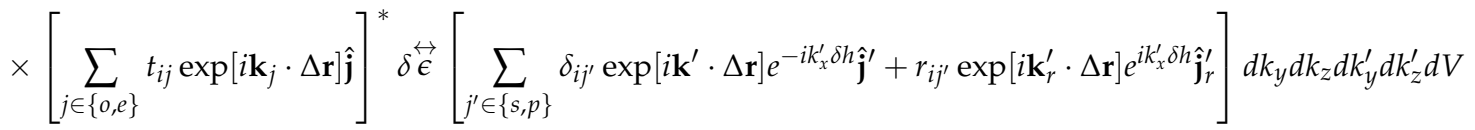

where $i=s, p$ for $v=\mathrm{TE}$, TM as previously discussed. Rearranging the order of integration, the integrals over $y$ and $z$ can be evaluated immediately by noting that $\int_{-\infty}^{\infty} \exp \left[i\left(k_{y}^{\prime}-k_{y}\right) y\right] d y=2 \pi \delta\left(k_{y}^{\prime}-k_{y}\right)$ and similarly for $k_{z}$. Consequently integration over $k_{y}^{\prime}$ and $k_{z}^{\prime}$ can 
also be simply performed yielding

$$
N_{\mathbf{v}}^{(1)}=4 \pi^{2}|\mathcal{A}(h)|^{2} \sum_{j \in\{0, e\}} \sum_{j^{\prime} \in\{s, p\}} \int_{x_{0}}^{x_{0}+\delta h} \iint_{-\infty}^{\infty}\left|\widetilde{E}_{\mathbf{v}}(\mathbf{k} ; h)\right|^{2} t_{i j}^{*}\left[\delta_{i j^{\prime}} e^{-\left(i k_{j, x}^{*}+\kappa\right) \Delta x} \hat{\mathbf{j}}^{*} \delta \overleftrightarrow{\leftrightarrow} \hat{\mathbf{j}}^{\prime}+r_{i j^{\prime}} e^{-\left(i k_{j, x}^{*}-\kappa\right) \Delta x} e^{-2 \kappa \delta h} \hat{\mathbf{j}}^{*} \delta \overleftrightarrow{\leftrightarrow} \hat{\mathbf{j}}_{r}^{\prime}\right] d k_{y} d k_{z} d x+O(\delta h)
$$

where we have expanded $\mathcal{A}(h+\delta h) \approx \mathcal{A}(h)+\delta h d \mathcal{A} / d h+\ldots$, used Eq. (20) to write $\widetilde{E}_{\mathbf{v}}\left(\mathbf{k}^{\prime} ; h+\delta h\right)=\widetilde{E}_{\mathbf{v}}\left(\mathbf{k}^{\prime} ; h\right) \exp [-\kappa \delta h]$ and made the substitution $-i k_{x} \approx \kappa$. The integration over $x$ can also be performed analytically such that we obtain

$$
\begin{aligned}
N_{\mathbf{v}}^{(1)} & =4 \pi^{2}|\mathcal{A}(h)|^{2} \sum_{j \in\{0, e\}} \sum_{j^{\prime} \in\{s, p\}} \iint_{-\infty}^{\infty} t_{i j}^{*}\left|\widetilde{E}_{\mathbf{v}}(\mathbf{k} ; h)\right|^{2} \\
& \times\left[\delta_{i j^{\prime}} \mathcal{X}_{j}^{+} \hat{\mathbf{j}}^{*} \delta \overleftrightarrow{\leftrightarrow} \hat{\mathbf{j}}^{\prime}+r_{i j} \mathcal{X}_{j}^{-} e^{-2 \kappa \delta h} \hat{\mathbf{j}}^{*} \delta \overleftrightarrow{\epsilon} \hat{\mathbf{j}}_{r}^{\prime}\right] d k_{y} d k_{z}+O(\delta h)
\end{aligned}
$$

where

$$
\begin{aligned}
\mathcal{X}_{j}^{ \pm} & =\int_{x_{0}}^{x_{0}+\delta h} \exp \left[-\left(i k_{j, x}^{*} \pm \kappa\right)\left(x-x_{0}\right)\right] d x \\
& =\frac{1-\exp \left[-\left(i k_{j, x}^{*} \pm \kappa\right) \delta h\right]}{i k_{j, x}^{*} \pm \kappa} .
\end{aligned}
$$

Evaluation of $N_{\mathbf{v}}^{(2)}$ : We begin by separating $N_{\mathbf{v}}^{(2)}$ into two distinct integrals: one over the hemisphere in the half-space $x \geq x_{0}$ (in the prism) and another over a hemisphere in the half-space $x<x_{0}$ (in the host medium), which we denote by $N_{\mathbf{v}}^{(2>)}+N_{\mathbf{v}}^{(2<)}$ respectively. In the dielectric substrate we can use Eq. (41) and write

$$
\begin{aligned}
\delta \mathbf{E}_{\mathbf{v}}(\mathbf{r})= & \mathcal{A}(h) \sum_{j \in\{0, e\}} \iint_{-\infty}^{\infty}\left[t_{i j} \widetilde{E}_{\mathbf{v}}(\mathbf{k} ; h) e^{i \mathbf{k}_{j} \cdot \Delta \mathbf{r}} \hat{\mathbf{j}}\right. \\
& \left.\times\left\{\exp \left[-\left(i k_{j, x}+\kappa\right) \delta h\right]-1\right\}\right] d k_{y} d k_{z}+O(\delta h) .
\end{aligned}
$$

We note, however, that we have chosen our integration surface such that $R_{V} \rightarrow \infty$ (see Appendix C). Consequently, through application of the method of stationary phase [62], Eq. (60) becomes

$$
\begin{aligned}
& \lim _{|\Delta \mathbf{r}| \rightarrow \infty} \delta \mathbf{E}_{\mathbf{v}}(\mathbf{r})=-2 \pi i \mathcal{A}(h) \\
& \times \sum_{j \in\{o, e\}} t_{i j} k_{j, x} \widetilde{E}_{\mathbf{v}}(\mathbf{k} ; h)\left\{e^{-\left(i k_{j, x}+\kappa\right) \delta h}-1\right\} \frac{e^{i k_{j} \Delta r}}{\Delta r} \hat{\mathbf{j}}+O(\delta h),
\end{aligned}
$$

where $\Delta r=|\Delta \mathbf{r}|$ and $k_{j}=\left|\mathbf{k}_{j}\right|$. Further noting $\omega \mu_{0} \widetilde{\mathbf{H}}_{\mathbf{v}}(\mathbf{k} ; h)=\mathbf{k} \times \widetilde{\mathbf{E}}_{\mathbf{v}}(\mathbf{k} ; h)$ and that in the far field $d \mathbf{S}=\Delta \hat{\mathbf{r}} d S=\hat{\mathbf{k}}_{j} d S$ we have for a fixed direction

$\lim _{|\Delta \mathbf{r}| \rightarrow \infty}\left[\delta \mathbf{E}_{\mathbf{v}}(\mathbf{r}) \times \mathbf{H}_{\mathbf{v}}^{*}(\mathbf{r})\right] \cdot \hat{\mathbf{k}}_{j}=\frac{4 \pi^{2}}{\omega \mu_{0}} \frac{|\mathcal{A}(h)|^{2}}{\Delta r^{2}} \sum_{j \in\{0, e\}} \sum_{j^{\prime} \in\{0, e\}} t_{i j} t_{i j^{\prime}}^{*} k_{j, x} k_{j^{\prime}, x}\left|\widetilde{\mathbf{E}}_{\mathbf{v}}(\mathbf{k} ; h)\right|^{2}\left\{\exp \left[-\left(i k_{j, x}+\kappa\right) \delta h\right]-1\right\} \hat{\mathbf{j}} \times\left(\mathbf{k}_{j^{\prime}} \times \hat{\mathbf{j}}^{\prime}\right) \cdot \hat{\mathbf{k}}_{j}+O(\delta h)$

where we have also restricted to propagating waves $\left(\operatorname{Im}\left[k_{j, x}\right]=\right.$ 0 ) since evanescent components do not contribute to the far field. Using the standard identity $\mathbf{a} \times(\mathbf{b} \times \mathbf{c})=\mathbf{b}(\mathbf{a} \cdot \mathbf{c})-\mathbf{c}(\mathbf{a} \cdot \mathbf{b})$ yields

$$
\hat{\mathbf{j}} \times\left(\mathbf{k}_{j^{\prime}} \times \hat{\mathbf{j}}^{\prime}\right) \cdot \hat{\mathbf{k}}_{j}=\delta_{j j^{\prime}} k_{j}\left[1-\left(\hat{\mathbf{j}} \cdot \hat{\mathbf{k}}_{j}\right)^{2}\right],
$$

where $\hat{\mathbf{o}} \cdot \hat{\mathbf{k}}_{j}=0$ but $\hat{\mathbf{e}} \cdot \hat{\mathbf{k}}_{j} \neq 0$ due to the anisotropic nature of the substrate. It can easily be shown that $\lim _{|\Delta \mathbf{r}| \rightarrow \infty}\left[\delta \mathbf{E}_{\mathbf{v}}(\mathbf{r}) \times\right.$ $\left.\mathbf{H}_{\mathbf{v}}^{*}(\mathbf{r})\right] \cdot \hat{\mathbf{k}}_{j}=\lim _{|\Delta \mathbf{r}| \rightarrow \infty}\left[\mathbf{E}_{\mathbf{v}}^{*}(\mathbf{r}) \times \delta \mathbf{H}_{\mathbf{v}}(\mathbf{r})\right] \cdot \hat{\mathbf{k}}_{j}$, and that the surface element is given by $d S=\Delta r^{2} \sin \vartheta d \vartheta d \phi=$ $\Delta r^{2} d k_{y} d k_{z} /\left(k_{j} k_{j, x}\right)$, whereby it follows that

$$
\begin{aligned}
& N_{\mathbf{v}}^{(2>)}=\frac{8 \pi^{2}}{\omega \mu}|\mathcal{A}(h)|^{2} \sum_{j \in\{0, e\}} \iint_{\operatorname{Im}\left[k_{j, x}\right]=0}\left[k_{j, x}\left|t_{i j}\right|^{2}\left|\widetilde{\mathbf{E}}_{\mathbf{v}}(\mathbf{k} ; h)\right|^{2}\right. \\
& \left.\quad \times\left\{e^{-\left(i k_{j, x}+\kappa\right) \delta h}-1\right\}\left\{1-\left(\hat{\mathbf{j}} \cdot \hat{\mathbf{k}}_{j}\right)^{2}\right\}\right] d k_{y} d k_{z}+O(\delta h) .
\end{aligned}
$$

In the half space $x<x_{0}$ a similar analysis can be performed as that presented for $x \geq x_{0}$. Critically, for large resonators the majority of the plane wave components in the medium surrounding the resonator are evanescent and do not contribute in the far field. From the law of reflection it also follows that the reflected plane wave components of the WGM are also evanescent in nature, such that we can conclude that $N_{\mathbf{v}}^{(2<)} \approx 0$.

Complex resonance shifts: Upon substituting Eqs. (54), (57) and (64) into Eq. (47) we note that the $|\mathcal{A}(h)|^{2}$ factors cancel from the leading terms in both the numerator and denominator. Upon taking the limit in Eq. (51) we thus find

$$
\frac{d \bar{\omega}}{d h}=-\frac{1}{U_{\mathbf{v}}^{\text {res }}}\left[\bar{\omega} \mathcal{N}_{\mathbf{v}}^{(1)}(h)+i \mathcal{N}_{\mathbf{v}}^{(2)}(h)\right]
$$

where

$$
\begin{aligned}
& \mathcal{N}_{\mathbf{v}}^{(1)}(h)=\pi^{2} \sum_{j \in\{0, e\}} \sum_{j^{\prime} \in\{s, p\}} \iint_{-\infty}^{\infty} t_{i j}^{*}\left|\widetilde{E}_{\mathbf{v}}(\mathbf{k} ; h)\right|^{2} \times\left[\delta_{i j^{\prime}} \hat{\mathbf{j}}^{*} \delta \overleftrightarrow{\epsilon} \hat{\mathbf{j}}^{\prime}+r_{i j} \hat{\mathbf{j}}^{*} \delta \overleftrightarrow{\leftrightarrow} \hat{\mathbf{j}}_{r}^{\prime}\right] d k_{y} d k_{z} \quad \text { (66) } \\
& \mathcal{N}_{\mathbf{v}}^{(2)}(h)=-\frac{2 \pi^{2}}{\omega \mu} \sum_{j \in\{0, e\}} \iint_{\operatorname{Im}\left[k_{j, x}\right]=0} k_{j, x}\left(i k_{j, x}+\kappa\right)\left|t_{i j}\right|^{2}\left|\widetilde{\mathbf{E}}_{\mathbf{v}}(\mathbf{k} ; h)\right|^{2} \\
& \times\left[1-\left(\hat{\mathbf{j}} \cdot \hat{\mathbf{k}}_{j}\right)^{2}\right] d k_{y} d k_{z} \cdot \mathbf{( 6 7 )}
\end{aligned}
$$



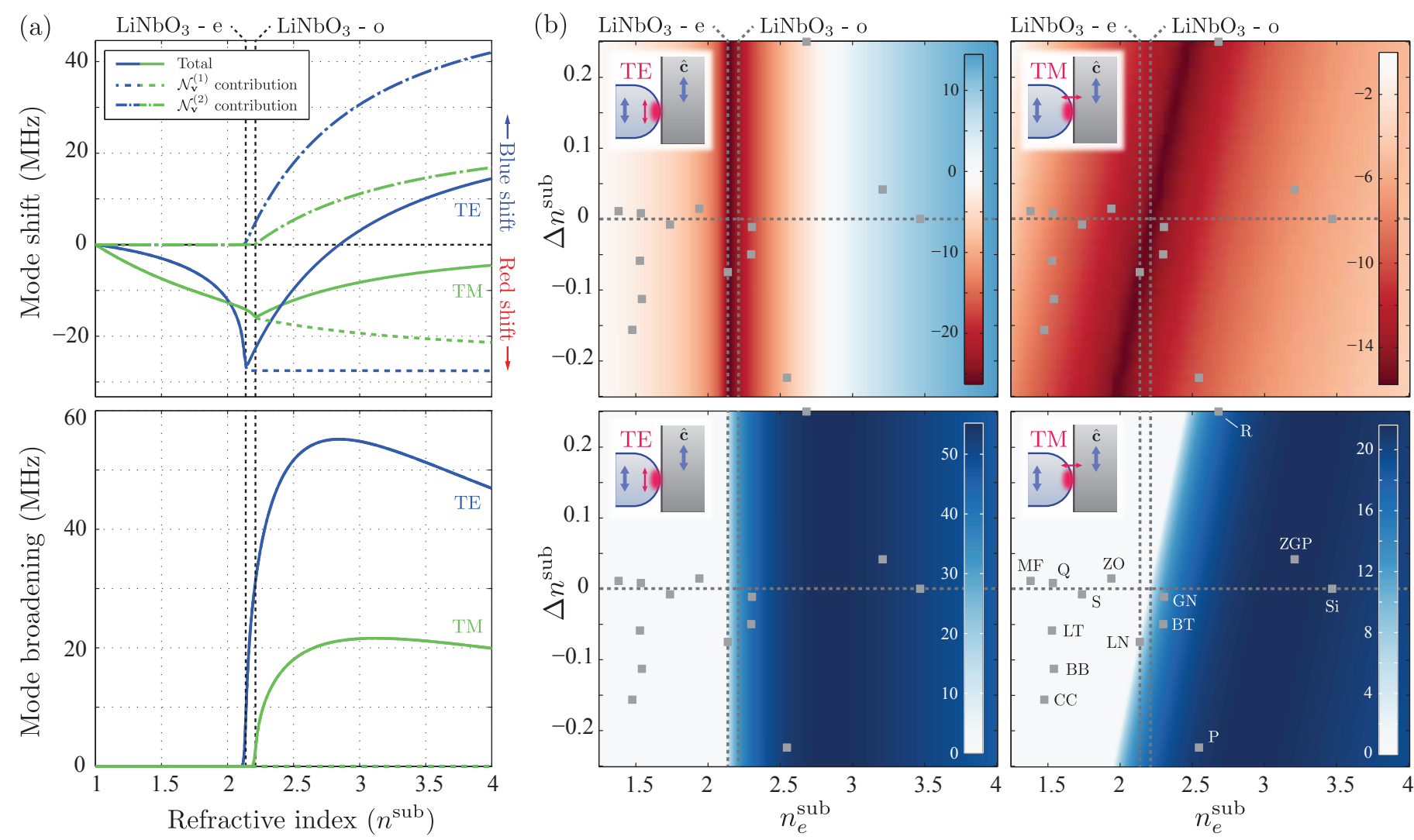

Fig. 3. Effects of substrate birefringence: (a) Dependence of the resonance shift $\Delta \omega_{\mathbf{v}} /(2 \pi)$ (top) and mode broadening $\Delta \gamma_{\mathbf{v}} /(2 \pi)$ (bottom) in MHz of TE (blue) and TM (green) WGMs supported in a $\mathrm{LiNbO}_{3}$ resonator induced by bringing an isotropic substrate of varying refractive index $n^{\text {sub }}$ into contact. Total resonance modifications (solid lines) derive from both a near field material polarisation $\mathcal{N}_{\mathbf{v}}^{(1)}$ (dashed) and a far field radiative $\mathcal{N}_{\mathbf{v}}^{(2)}$ (dash-dotted) contribution. (b) Variation of the (top) mode shift (in MHz) and (bottom) broadening (in MHz) of TE (left) and TM (right) WGMs with substrate birefringence $\Delta n^{\text {sub }}=n_{e}^{\text {sub }}-n_{o}^{\text {sub }}$. The optic axis of the substrate is assumed to be parallel to that of the resonator as depicted in the insets. Grey squares indicate material properties of some common (birefringent) substrates: magnesium fluoride, $\mathrm{MgF}_{2}$ (labelled $\mathrm{MF}$ ); crystal quartz, $\mathrm{SiO}_{2}(\mathrm{Q})$; sapphire, $\mathrm{Al}_{2} \mathrm{O}_{3}(\mathrm{~S})$; lithium tetraborate, $\mathrm{Li}_{2} \mathrm{~B}_{4} \mathrm{O}_{7}(\mathrm{LT})$; barium borate, $\mathrm{BaB}_{2} \mathrm{O}_{4}(\mathrm{BB})$; lithium niobate, $\mathrm{LiNbO}_{3}(\mathrm{LN})$; calcite, $\mathrm{CaCO}_{3}(\mathrm{C})$; zinc oxide, $\mathrm{ZnO}$ (ZO); gallium nitride, $\mathrm{GaN}$ (GN); barium titanate, $\mathrm{BaTiO}_{3}(\mathrm{BT})$; proustite, $\mathrm{Ag}_{3} \mathrm{AsS}_{3}(\mathrm{P})$; zinc germanium phosphide $\mathrm{ZnGeP}$ (ZGP); rutile, $\mathrm{TiO}_{2}(\mathrm{R})$; and silicon, $\mathrm{Si}(\mathrm{Si})$. Dashed vertical lines depict the ordinary and extraordinary refractive indices of the resonator. Refractive index data were taken from [63-74].

Finally, noting $\left|\widetilde{E}_{\mathbf{v}}(\mathbf{k} ; h)\right|^{2}=\left|\widetilde{E}_{\mathbf{v}}(\mathbf{k} ; 0)\right|^{2} \exp [-2 \kappa h]$, we can evaluate Eq. (52) to obtain

$$
\Delta \bar{\omega}_{\mathbf{v}}(h)=\frac{\exp [-2 \kappa h]}{2 \kappa U_{\mathbf{v}}^{\text {res }}}\left[\bar{\omega} \mathcal{N}_{\mathbf{v}}^{(1)}(0)+i \mathcal{N}_{\mathbf{v}}^{(2)}(0)\right]
$$

from which the shift in the (real) resonance frequency and the change in the line width follow as $\Delta \omega_{\mathbf{v}}=\operatorname{Re}\left[\Delta \bar{\omega}_{\mathbf{v}}\right]$ and $\Delta \gamma_{\mathbf{v}}=$ $-2 \operatorname{Im}\left[\Delta \bar{\omega}_{\mathbf{v}}\right]$ respectively. Eq. (68) constitutes the key result of this article and will form the basis of the remaining analysis.

\section{NUMERICAL RESULTS AND DISCUSSION}

To study the resonance perturbations induced by a (uniaxial) dielectric substrate we now apply Eq. (68) to a number of scenarios. In all calculations we consider a lithium niobate $\left(\mathrm{LiNbO}_{3}\right)$ resonator supporting WGMs at $\lambda \approx 1550 \mathrm{~nm}$, whereby the ordinary and extraordinary refractive indices are given by $n_{o}=2.213$ and $n_{e}=2.138$ respectively [63]. Furthermore, the major and minor radii of the resonator are taken to be $R=2.1 \mathrm{~mm}$ and $P=R / 9$ respectively and the optic axis of the resonator is assumed to be parallel to its axis of rotation (i.e. $z$-cut). Accordingly, the fundamental $(p=0, q=1) \mathrm{TE}$ and TM resonances at $\lambda \approx 1550 \mathrm{~nm}$, as found using the dispersion relation given in [43], are of order $m=18152$ and 18790 respectively. The associated amplitude decay lengths are $\kappa^{-1} \approx 130 \mathrm{~nm}$ and $125 \mathrm{~nm}$.

\section{A. Anomalous radiative shifts}

Before considering the more general scenario of uniaxial substrates we first analyse the resonance perturbations induced by an isotropic substrate. The solid curves in Figure 3(a) show the calculated resonance shift $\Delta \omega_{\mathrm{v}} /(2 \pi)$ (top plot) and mode broadening $\Delta \gamma_{\mathbf{v}} /(2 \pi)$ (bottom) in $\mathrm{MHz}$ that result when a dielectric substrate of varying refractive index, $n^{\text {sub }}$, is brought from infinity into contact with the resonator $(h=0)$. As described by Eq. (68) the total complex frequency shift derives from two distinct contributions. Specifically, the $\mathcal{N}_{\mathbf{v}}^{(1)}$ term in Eq. (68) relates to work done in polarising the dielectric and to material absorption therein (the latter is assumed to be zero in all calculations). Moreover, the $\mathcal{N}_{\mathbf{v}}^{(1)}$ term is of identical form to the usual pertur- 
bation integral used to study reactive resonance shifts [33, 61]. The second $\mathcal{N}_{\mathbf{v}}^{(2)}$ term, however, is usually omitted in conventional treatments of cavity perturbations but is required when describing open cavities and relates to coupling of the WGM into the far field [44], which can be further facilitated by introduction of the substrate. The individual contributions from these terms are also indicated in Figure 3(a) by the dashed and dot-dashed curves respectively.

As the refractive index of the substrate increases from unity, it is seen from Figure 3(a) that the WGM resonance frequency is red-shifted. For small refractive indices, the transmitted field in the substrate is purely evanescent, such that the red-shift originates solely from the work done generating a material polarisation, which can equivalently be considered as an increase in the effective refractive index, and hence optical path length, of the WGM propagating in the resonator. Accordingly, no additional radiative losses are introduced into the system and no mode broadening is seen. As $n^{\text {sub }}$ increases further so too does the magnitude of the red shift (due to the larger resulting index contrast), until the refractive index of the substrate is approximately equal to the effective refractive index of the unperturbed WGMs i.e. $n^{\text {sub }} \approx n^{\text {eff }}$ (albeit not exactly due to the non-zero width of the WGM angular spectrum). Once this condition is met a maximum red shift results. We note that by virtue of the anisotropy of the lithium niobate resonator assumed in our calculations, the effective refractive index for TE and TM WGMs differs ( $n^{\text {eff }} \approx n_{e}$ and $n_{o}$ respectively) and hence so too does the position of the maximum red shift.

For yet larger values of $n^{\text {sub }}$ the magnitude of the red shift starts to decrease. Physically, the qualitative change in the frequency shift arises because when $n^{\text {sub }} \gtrsim n^{\text {eff }}$ the field transmitted into the substrate is no longer purely evanescent, but can contain significant (or even only) propagating components. Consequently, the total shift derives from competition between the red shifts arising from the $\mathcal{N}_{\mathbf{v}}^{(1)}$ term and blue shifts from the radiative $\mathcal{N}_{\mathbf{v}}^{(2)}$ term. For (predominantly s polarised) TE modes, the $\mathcal{N}_{\mathbf{v}}^{(1)}$ contribution is constant regardless of substrate refractive index since the change in the material polarisability is precisely compensated for by the change in transmission into and reflection from the substrate. The differing dependence of the Fresnel coefficients on the substrate refractive index for incident $p$ polarised TM modes, however, means that the resulting red shift arising from material polarisation weakly increases with $n^{\text {sub }}$. In contrast, the $\mathcal{N}_{\mathbf{v}}^{(2)}$ resonance shift, which arises from interference between the WGM and the additional radiated field induced by shifting the position of the substrate (or equivalently a back action from the radiation continuum [44]) is towards higher frequencies. Ultimately when the substrate refractive index is large enough, the radiation interaction can dominate the resonance shift giving rise to an anomalous net blue shift of the WGM relative to the case when no substrate is present. This transition from a red to blue shift occurs at much lower substrate refractive indices for TE modes than for TM modes due to the differing behaviour of $\mathcal{N}_{\mathbf{v}}^{(1)}$ for $n^{\text {sub }} \gtrsim n^{\text {eff }}$. Practically, the substrate refractive index required to observe blue shifts of TM modes is unphysically large within the optical domain. Although we shall not consider this case in any detail in this article, it is worth noting that WGM blue shifts can also be observed when the refractive index of the medium surrounding the resonator is greater than that of the substrate as follows from the $\delta \overleftrightarrow{\epsilon}$ dependence of Eq. (66), or equivalently the change in sign of the material polarisability as follows from the Clausius-Mossotti relation.

As noted above, when the refractive index of the substrate is larger than the effective refractive index seen by the WGM in the unperturbed resonator, light couples to radiative, i.e. propagating waves in the substrate. Energy is carried by these propagating waves to infinity such that they constitute a loss mechanism. Accordingly, once coupling of the WGM to these propagating modes is possible, the line width of the resonance increases as seen in Figure 3(a). Briefly noting that the relative fraction of the mode energy that is contained exterior to the resonator (Eq. (9)) is smaller for TM modes as compared to TE modes it would be expected that the resulting mode broadening is also smaller for TM modes. This expectation is indeed borne out in calculations as apparent from Figure 3(a). Furthermore, we observe that as we increase the substrate refractive index, the coupling rate increases to a maximum value, before slowly decreasing at larger refractive indices. This behaviour can be understood by first noting that the Fresnel transmission coefficients decrease with increasing substrate refractive index (i.e. there is stronger reflection at the interface). Larger substrate refractive index, however, also implies that the $k_{x}$ component of the transmitted wavevector increases (i.e. the transmitted wave propagates at a smaller angle to the surface normal), such that the projection factor appearing when considering energy conservation at the interface also increases. These two opposing effects give rise to the maximum seen in Figure 3(a).

Extension of the calculations to the case of uniaxial substrates shows similar trends as to the isotropic case, as shown in Figure 3(b). Specifically, the resonance shift $\Delta \omega_{\mathbf{v}} /(2 \pi)$ (top row) and mode broadening $\Delta \gamma_{\mathbf{v}} /(2 \pi)$ (bottom row) in $\mathrm{MHz}$ are shown as a function of the extraordinary refractive index of the substrate $n_{e}^{\text {sub }}$ and the substrate birefringence $\Delta n^{\text {sub }}=$ $n_{e}^{\text {sub }}-n_{o}^{\text {sub }}$. The optic axis of the substrate is assumed to be parallel to that of the resonator, i.e. $\hat{\mathbf{c}}=\hat{\mathbf{z}}$. Although a weak dependence of the TE shift and broadening on the substrate birefringence results from the cross-polarisation mixing described in Eqs. (23)-(24), this effect is found to be several orders of magnitude smaller than the co-polarised terms, such that only variation of the resonance properties with the substrate extraordinary index, and not $\Delta n^{\text {sub }}$, is seen in Figure 3(b), mirroring that found for the isotropic case. When $\hat{\mathbf{c}}=\hat{\mathbf{z}}$, TM modes also effectively see an isotropic substrate with refractive index $n_{0}^{\text {sub }}$. As such the resulting functional dependence of the shifts and broadening exhibits a linear displacement with substrate birefringence for fixed $n_{e}^{\text {sub }}$. This effect could, for example, hence be exploited for differential tuning of TE and TM WGMs if the substrate has a large linear electro-optic coefficient and the resonator itself does not show strong electro-optic effects.

Anisotropy of the substrate also plays an important role in dictating the WGM shifts when the optic axis is varied, as shown in Figure 4. Letting $\hat{\mathbf{c}}=\left[\sin \alpha_{c} \cos \beta_{c}, \sin \alpha_{c} \sin \beta_{c}, \cos \alpha_{c}\right]$, where $\alpha_{c}$ and $\beta_{c}$ are the polar and azimuthal angles of the optic axis (see Figure 4(a)), we have calculated the resulting resonance shift when a magnesium fluoride $\left(\mathrm{MgF}_{2}: n_{e}=1.382, n_{0}=1.371\right)$, lithium niobate $\left(\mathrm{LiNbO}_{3}: n_{e}=2.138, n_{0}=2.213\right)$ or rutile $\left(\mathrm{TiO}_{2}\right.$ : $\left.n_{e}=2.683, n_{0}=2.432\right)$ prism is brought into contact with the $\mathrm{LiNbO}_{3}$ resonator from infinity, as a function of $\left(\alpha_{c}, \beta_{c}\right)$. These three specific materials were selected since both the ordinary and extraordinary refractive index of $\mathrm{MgF}_{2}\left(\mathrm{TiO}_{2}\right)$ are smaller (larger) than that of the $\mathrm{LiNbO}_{3}$ resonator, whereas choice of a $\mathrm{LiNbO}_{3}$ substrate also allows the intermediate regime to be analysed. Numerical results are shown in Figure 4(b). 


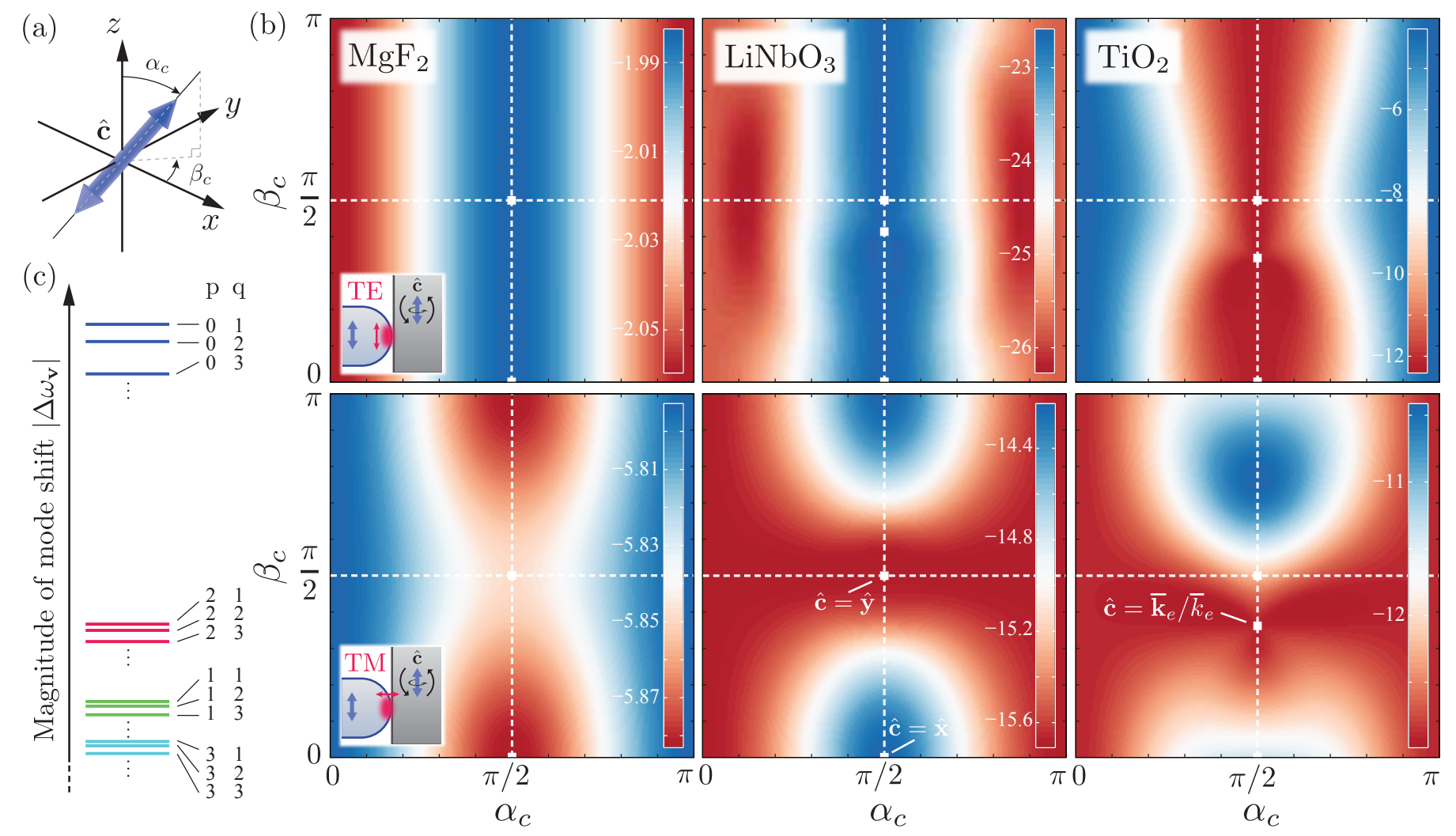

Fig. 4. Optic axis and modal dependence of resonance shifts: (a) Coordinate system defining orientation of the optic axis $\hat{c}$ of the substrate. (b) Resonance shifts $\Delta \omega_{\mathbf{v}} /(2 \pi)$ (in $\mathrm{MHz}$ ) induced in TE (top row) and TM (bottom) WGMs upon bringing a magnesium fluoride, $\mathrm{MgF}_{2}$ (left column); lithium niobate, $\mathrm{LiNbO}_{3}$ (middle); or rutile, $\mathrm{TiO}_{2}$ substrate into contact with a LiNbO 3 resonator, as a function of the orientation of the substrate optic axis $\hat{\mathbf{c}}=\left[\sin \alpha_{c} \cos \beta_{c}, \sin \alpha_{c} \sin \beta_{c}, \cos \alpha_{c}\right]$. Note that for the substrate refractive indices considered only red shifts are predicted. (c) Schematic of the typical hierarchy of resonances shifts for WGMs of fixed ( $m, v$ ) but differing polar and radial index $(p, q)$. A similar hierarchy is also seen for modes of (approximately) fixed wavelength, but differing $(m, p, q)$.

Considering first the $\mathrm{MgF}_{2}$ substrate, we note that since all waves transmitted into the substrate are evanescent the resonance shift originates only from the work done in polarising the medium as before. Accordingly, since $\mathrm{MgF}_{2}$ is positively birefringent $\left(n_{e}^{\text {sub }}>n_{o}^{\text {sub }}\right)$, if the optic axis is rotated from the $\hat{\mathbf{z}}$ axis towards the $x-y$ plane $\left(\alpha_{c}\right.$ is increased) the magnitude of the shift of the TE mode decreases because the effective refractive index seen by the WGM also decreases. Conversely, since the TM WGM is predominantly polarised in the $x-y$ plane a larger magnitude resonance shift results under the same rotation of $\hat{\mathbf{c}}$. Rotation of the optic axis around the $\hat{\mathbf{z}}$ axis through an increase of $\beta_{c}$, also gives rise to a modulation in the resonance shift of the TM mode through the same mechanism. The largest magnitude shift therefore results when the optic axis is perpendicular to the field component with the greatest amplitude in the near field. For TM modes this corresponds to $\beta_{c}=0$ i.e. the radial direction at the contact point between the resonator. No variation in the TE resonance frequency with $\beta_{c}$ is however seen, since the mode is polarised along the axis of rotation. For a negatively birefringent substrate $\left(n_{e}^{\text {sub }}<n_{o}^{\text {sub }}\right)$, such as barium borate, these trends would be reversed, e.g. the TE mode would experience smaller red shifts as $\alpha_{c}$ was increased to $\pi / 2$ as opposed to larger shifts. We also note that the modulation of the resonance shift due to the variation of the material polarisability increases with the birefringence of the substrate. A rough order of magnitude estimate of the relative modulation can be obtained by considering the relative change in the Clausius-Mossotti polarisability when evaluated using the ordinary and extraordinary refractive indices. Typically, for the materials considered in this work variation of only a few percent (or equivalently $\lesssim 1 \mathrm{MHz}$ ) were seen as also evidenced by the data in Figure 4.

Although the dependence of the resonance shifts for WGMs resulting from the presence of $\mathrm{a} \mathrm{TiO}_{2}$ substrate are qualitatively similar to those for a $\mathrm{MgF}_{2}$ substrate, the physical origin is quite different. This is apparent since both $\mathrm{TiO}_{2}$ and $\mathrm{MgF}_{2}$ are positively birefringent materials, yet the observed trends are in opposition to each other. Since both the ordinary and extraordinary refractive indices of $\mathrm{TiO}_{2}$ are larger than that of the resonator, the field in the substrate is composed of propagating waves. As was discussed above, as the optic axis of the substrate is varied, so the effective refractive index of the substrate seen by the WGM also varies, however, with reference to Figure 3(a), when in the propagating transmitted wave regime $\left(n_{e, 0}^{\text {sub }} \gtrsim n^{\text {eff }}\right)$ the relative change in the resonance frequency is dominated by the radiative shift term $\left(\mathcal{N}_{\mathbf{v}}^{(2)}\right)$. Mathematically speaking, since $\left|\partial \operatorname{Re}\left[i \mathcal{N}_{\mathbf{v}}^{(2)}\right] / \partial \hat{\mathbf{c}}\right|>\left|\partial \operatorname{Re}\left[\bar{\omega} \mathcal{N}_{\mathbf{v}}^{(1)}\right] / \partial \hat{\mathbf{c}}\right|$ when $n_{e, o}^{\text {sub }} \gtrsim n^{\text {eff }}$, the optic axis dependence of the resonance shift is dominated by the radiative component of the Bethe-Schwinger equation. Importantly, negative frequency shifts can still be observed (as in 
the $\mathrm{TiO}_{2}$ data in Figure $\left.4(\mathrm{~b})\right)$ if $\left|\operatorname{Re}\left[\bar{\omega} \mathcal{N}_{\mathbf{v}}^{(1)}\right]\right|>\left|\operatorname{Re}\left[i \mathcal{N}_{\mathbf{v}}^{(2)}\right]\right|$. The modulation of the resonance frequency from the radiative interaction corresponds to several tens of percent, or equivalently to $\lesssim 10 \mathrm{MHz}$, depending on the material. Considering $\mathrm{TiO}_{2}$ for definiteness, we note that as the optic axis is rotated from the $\hat{\mathbf{z}}$ axis towards the $x-y$ plane ( $\alpha_{c}$ is increased) such that the effective refractive index seen by a TE mode decreases, coupling of the WGM to the far field is reduced due to lower transmission. Consequently the corresponding blue shift from the radiative back action is also reduced such that in total a larger red shift is observed (see Figure 4(b)). The converse again holds for TM modes. Interestingly, in the frequency shifts calculated for rutile a weakly singular feature can also be discerned, which occurs when the central wavevector of the output extraordinary beam is parallel to the optic axis of the substrate. This special case will be discussed further below.

When a $\mathrm{LiNbO}_{3}$ substrate is used to tune the resonance frequency a mixed behaviour is seen. Due to the choice of optic axis of the resonator, the refractive index experienced by a TE WGM in the substrate is always larger than the effective refractive index in the resonator. Accordingly a propagating field is transmitted into the substrate and the dependence on the optic axis is dictated by the variation in $\mathcal{N}_{\mathbf{v}}^{(2)}$ as was the case for $\mathrm{TiO}_{2}$ (we note the opposing trends between $\mathrm{LiNbO}_{3}$ and $\mathrm{TiO}_{2}$ is a result of the opposite signature of the birefringence). TM modes, however, couple predominantly to evanescent waves in the substrate and hence the dependence on the optic axis is governed by $\mathcal{N}_{\mathbf{v}}^{(1)}$ as was found with $\mathrm{MgF}_{2}$.

In the calculations presented thus far we have only considered the resonance shifts induced in WGMs for which $p=0$ and $q=1$. Naturally, a quantitative difference in the induced shift is seen for modes of differing polar and radial order (but fixed $m$ and $v$ ). Under typical conditions the hierarchy of shifts is that shown schematically in Figure 4(c). Specifically, as the radial order increases, so the magnitude of the shift falls slightly. Modes of differing polar order, however, form two separate ladders corresponding to modes of the same parity (i.e. odd or even symmetry). Within each individual ladder, smaller magnitudes shifts are seen for higher values of $p$ because for such higher order modes a greater proportion of the mode lies outside the Gaussian coupling window and hence does not interact significantly with the substrate. Deviations from the mode ordering shown in Figure 4(c) do, however, occur when the shifts are close to zero. It should also be noted that since modes of fixed $m$ have been considered here, modes of differing $(p, q)$ have different resonant frequencies. Nevertheless, if WGMs of approximately fixed wavelength are chosen (and $m$ varied accordingly) a similar ordering is also found.

\section{B. Selective coupling}

In addition to the resonance shifts discussed in the previous section, introduction of a substrate can give rise to coupling of the WGM into the far field and hence to mode broadening. Intuitively, this phenomenon can be understood since coupling to propagating waves in the substrate introduces additional loss channels for the WGM, yielding a shorter resonance lifetime. Since propagating waves are only excited when the effective refractive index of the substrate is larger than that seen by the WGM in the resonator, we now consider only the effect of either a $\mathrm{LiNbO}_{3}$ or $\mathrm{TiO}_{2}$ substrate. Figure 5(a) shows the mode broadening determined using Eq. (68) as a function of the orientation of the optic axis of the substrate. Anisotropy of the substrate implies that a given WGM can couple to both ordinary and extraordinary polarised waves in the substrate, such that the mode broadening results from the net effect of both channels. Accordingly, we also show the individual contributions from the ordinary and extraordinary beams in the leftmost columns of Figure 5(a).

Initially considering a $\mathrm{TiO}_{2}$ substrate, we again note that as $\alpha_{c}$ is increased to $\pi / 2$ the refractive index seen by TE (s polarised) WGMs decreases. Lower transmission into the far field hence results such that the mode broadening is reduced. Moreover, as the optic axis is rotated the ordinary and extraordinary polarisation vectors also rotate, such that the ordinary and extraordinary waves play complementary roles. For instance, when $\hat{\mathbf{c}}=\hat{\mathbf{z}}$ the TE mode couples to only extraordinary waves in the substrate, however, if $\alpha_{c}=\pi / 2$ a strong ordinary component can also be excited. Proportionally, the relative strength with which TE modes couple to ordinary waves varies with $\beta_{c}$. A weakly singular point is seen in the plots of Figure 5(a) corresponding to the case where $\hat{\mathbf{c}}=\overline{\mathbf{k}}_{e} /\left|\overline{\mathbf{k}}_{e}\right|$. When this condition is meet there is a minimum (maximum) in the refractive index experienced by the central angular component of the extraordinary wave when propagating in a positively (negatively) birefringent material. Furthermore, the polarisation vector $\hat{\mathbf{e}}$ is undefined for this angular component, such that a plane wave component propagating with wavevector parallel to $\hat{c}$ does so as a pure ordinarily polarised wave. Nevertheless, for an out-coupled WGM the extraordinary beam still carries a finite (albeit relatively small) amount of power to the far field due to the finite width of the incident angular spectrum. For an isotropic substrate, the ordinary and extraordinary wave vectors are equal and the complementarity of the corresponding beams is exact in the sense that a decrease in the energy carried by the ordinary beam resulting from a change in $\hat{\mathbf{c}}$, is compensated by an increase of equal magnitude in that of the extraordinary wave. Quantitative differences arise for anisotropic substrates however (as seen in Figure 5(a)), due to the differing dependence of the generalised Fresnel transmission coefficients and since $\mathbf{k}_{o}$ and $\mathbf{k}_{e}$ are not parallel. Similar, albeit reversed arguments apply for a TM WGM coupled to a $\mathrm{TiO}_{2}$ substrate. When broadening of WGMs of differing polar and radial orders is considered, a similar hierarchy to that for resonance shifts is again found (see Figure 4(c)).

When a $\mathrm{LiNbO}_{3}$ substrate is brought into close proximity to a WGM resonator, markedly different behaviour can be seen. As noted in the previous section, a TE WGM always couples to propagating waves in the substrate yielding similar behaviour to that seen for a $\mathrm{TiO}_{2}$ substrate (allowing for differences arising from the sign of the birefringence of each material). In contrast a TM mode mostly couples to evanescent waves except for within a restricted range of $\hat{c}$ corresponding to that bounded by the grey dashed circle in Figure 5(a). Only relative weak broadening of the TM mode hence results. Dependence of the partial mode broadening, originating from the ordinary and extraordinary waves individually, on $\hat{\mathbf{c}}$ is, in contrast to the propagating wave case, dominated by the non-radiative component $\left(\mathcal{N}_{\mathbf{v}}^{(1)}\right)$ of the Bethe-Schwinger equation since the radiative losses are so low. Counter-intuitively it is seen that an apparent mode narrowing can occur in either the ordinary or extraordinary channel. Importantly net mode broadening of TM WGMs perturbed by a $\mathrm{LiNbO}_{3}$ substrate is nevertheless governed by the radiative loss $\left(\mathcal{N}_{\mathbf{v}}^{(2)}\right)$ term as per physical intuition, since the near field $\left(\mathcal{N}_{\mathbf{v}}^{(1)}\right)$ contributions from the ordinary and extraordinary waves 
(a)
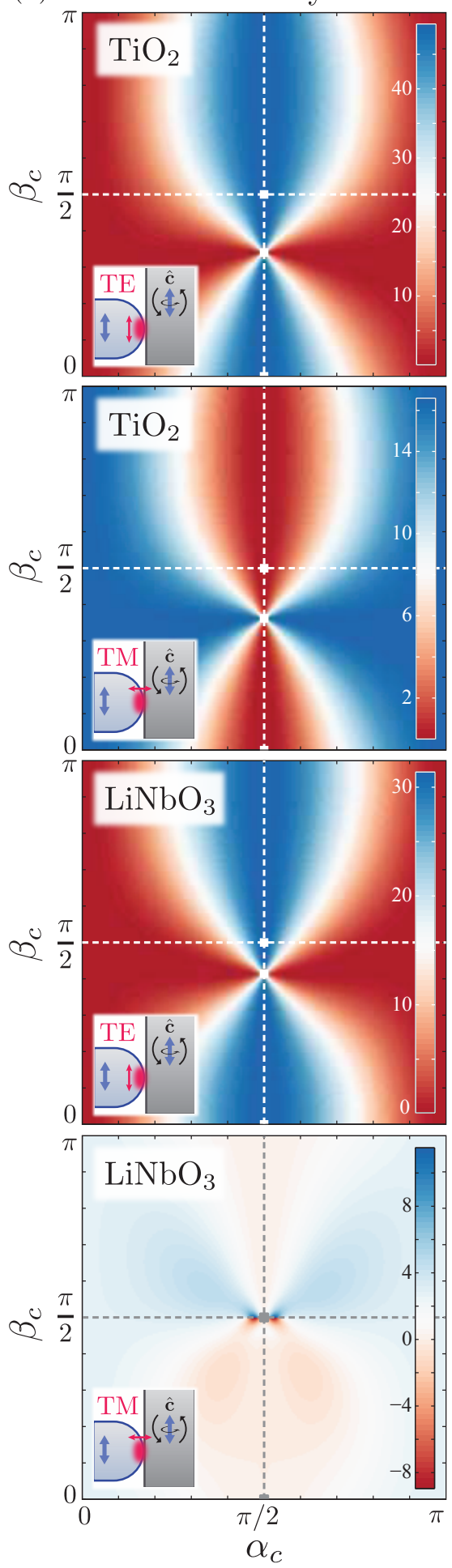

Extraordinary
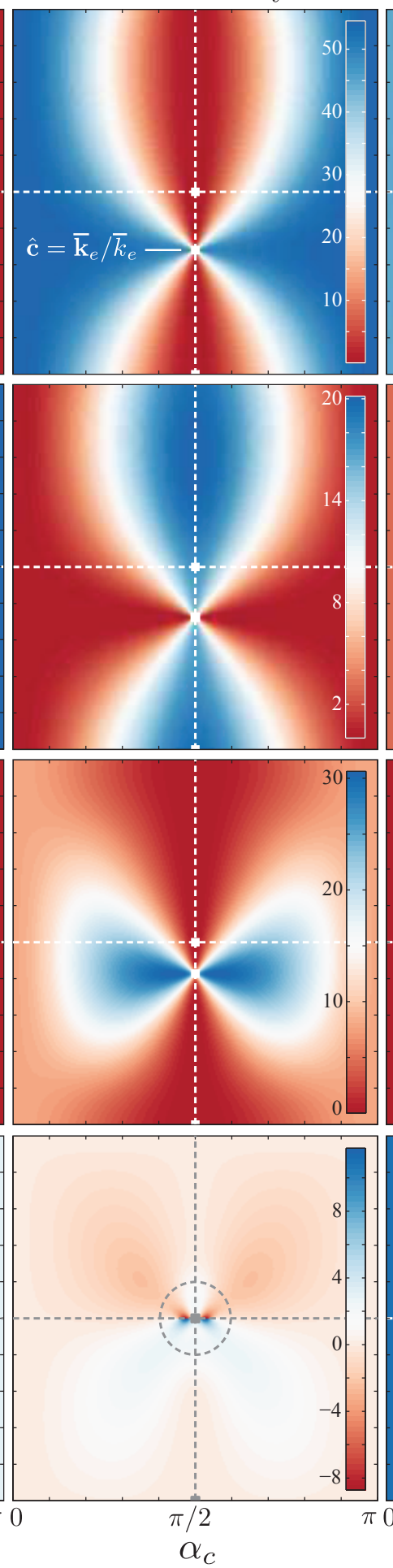

Total
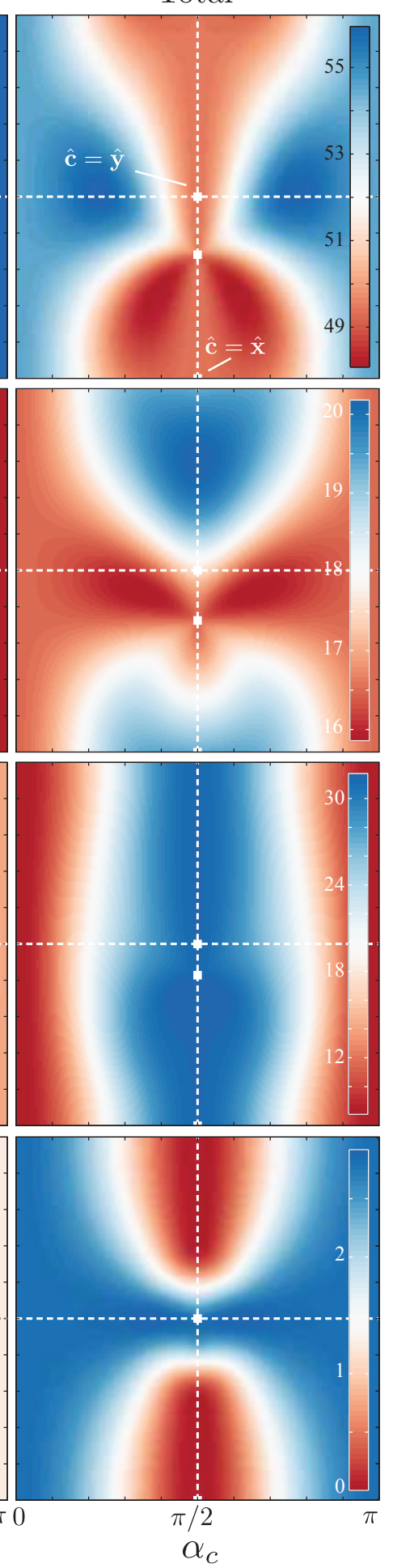

(b)

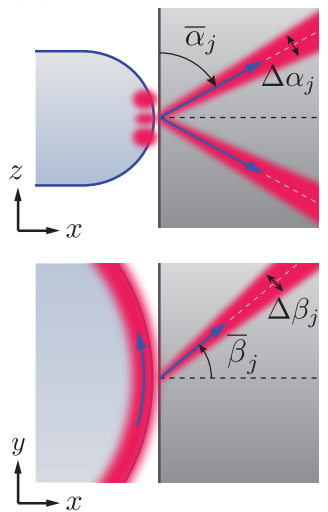

(c) $\widetilde{E}_{\mathbf{v}}(\mathbf{k} ; 0)$

$\mathrm{p}=0$

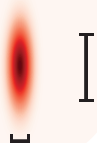

$\mathrm{p}=1$

$\mathrm{p}=2$

$\mathrm{p}=3$

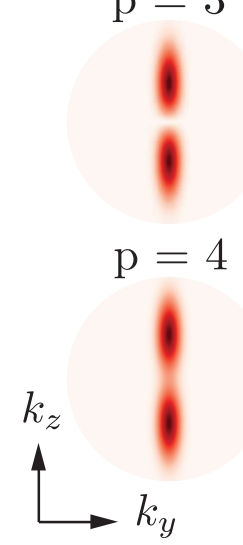

Fig. 5. Mode broadening and out-coupled beam parameters: (a) Mode broadening $\Delta \gamma_{\mathbf{v}} /(2 \pi)$ (in MHz) of TE (first and third rows) and TM (second and fourth rows) WGMs upon bringing a $\mathrm{TiO}_{2}$ (top two rows) or $\mathrm{LiNbO}_{3}$ (bottom two rows) substrate into contact with a $\mathrm{LiNbO}_{3}$ resonator, as a function of the orientation of the optic axis $\hat{c}$ of the substrate. The total mode broadening (right column) results from losses carried by an out coupled ordinary (left) and extraordinary (middle) beam. Note that apparent mode narrowing in the individual ordinary and extraordinary channels in the bottom row (arising from the $\mathcal{N}_{\mathbf{v}}^{(1)}$ term) is artificial since narrowing in one channel is compensated by an equal and opposite loss in the other, such that the net loss is dictated solely by $\mathcal{N}_{\mathbf{v}}^{(2)}$ and only mode broadening is seen. (b) Schematic defining the angular direction and angular width of propagating beams coupled into the substrate. (c) Scalar profiles $\widetilde{E}_{\mathbf{v}}(\mathbf{k}, 0)$ of out-coupled beams of different polar order $p$. Scale bars correspond to the FWHM of the fundamental $p=0$ mode in the $k_{y}$ and $k_{z}$ directions i.e. $2 \sqrt{2 \ln 2} / \Delta y$ and $2 \sqrt{2 \ln 2} / \Delta z$ respectively. 
are equal and opposite in magnitude and therefore precisely sum to zero. We briefly note, that the sudden transition from mode broadening to mode narrowing (and vice-versa) seen at $\beta_{c}=\pi / 2$ in the individual loss channels is an artifact of the definition of the (extra-)ordinary polarisation and wave vectors and is not of physical significance, especially considering there is no net effect from both channels. Given that a $\mathrm{LiNbO}_{3}$ substrate produces significant broadening of TE, but not TM, modes we note that this scenario corresponds to polarisationselective coupling of WGMs as has recently been experimentally demonstrated [42]. Maximum extinction ratios, that is to say the relative coupling rates, are achieved when $\hat{c}$ lies in the $x-y$ plane $\left(\alpha_{c}=\pi / 2\right)$, specifically along the $\hat{\mathbf{x}}$ axis $\left(\beta_{c}=0\right)$. In this case extinction ratios $\sim 1.6 \times 10^{4}$ (or equivalently $\sim 42 \mathrm{~dB}$ ) are predicted corresponding to a strong degree of selectivity.

\section{Geometric properties of out-coupled modes}

Finally, in this section we briefly consider the geometric properties of out coupled propagating ordinary and extraordinary beams in a uniaxial substrate when it is brought close to a WGM resonator. Specifically we seek expressions for the output angle of the beams and the angular widths as defined in Figure 5(b), which are important parameters in mode identification [75]. Similar considerations for the emission patterns of WGMs supported in spheroidal resonators coupled using isotropic prisms were given by Gorodetsky and Ilchenko [36], however, here we provide the corresponding expressions for a uniaxial prism. Most importantly, it should be noted that use of a uniaxial prism means that the angular components of the WGM each undergo double refraction, such that two beams are output in different directions and with differing polarisation state. Depending on the magnitude of the substrate birefringence and optic axis orientation, these two beams can, however, partially or completely overlap.

We begin by first writing the wavevector of a general angular component in the form $\mathbf{k}=k[\sin \alpha \cos \beta, \sin \alpha \sin \beta, \cos \alpha]$ (see Figure 1). Noting that the transverse wave components of each angular component are conserved across the substrate interface we can deduce the output angle in the $x-y$ plane $\left(\bar{\beta}_{j}\right)$ by considering $\bar{k}_{y}=k_{y r}=m / R=k_{j} \sin \bar{\alpha}_{j} \sin \bar{\beta}_{j}$ for $j \in\{o, e\}$. It immediately follows that

$$
\sin \bar{\beta}_{j}=\frac{m}{k_{j} R \sin \bar{\alpha}_{j}} .
$$

The output angles in the $z$ direction require a slightly more detailed analysis. Within the Gaussian window the scalar mode profile incident on the interface is an oscillatory function such that it follows that, except for the trivial case of $p=0$ for which $\bar{\alpha}_{j}=\pi / 2$, two distinct peaks located symmetrically about $k_{z}=0$ are expected in the far field (as shown in Figures 5(b) and (c)). As an approximation we first consider the $k_{z}$ dependence of the incident angular spectrum neglecting the effect of the Gaussian coupling window which can be easily shown to be given by the Hermite functions. To first order, the output beams correspond to the outermost peaks of the Hermite functions, which can be well approximated as

$$
\exp \left[-u^{2} / 2\right] H_{p}(u) \sim 2^{(2 p+1) / 4} \sqrt{\pi(p-1) !} \operatorname{Ai}[t]
$$

where $t=2^{1 / 2} p^{1 / 6}\left[(2 p+1)^{1 / 2}-u\right]$ and $u=P \Theta_{m} k_{z}$. Accord- ingly, the peaks are found at angles given by

$$
\cos \bar{\alpha}_{j} \approx \frac{1}{k_{j} P \Theta_{m}}\left[\sqrt{2 p+1}-\frac{\zeta_{1}^{\prime}}{2^{1 / 2} p^{1 / 6}}\right]
$$

where $\zeta_{1}^{\prime}=1.0188$ is the first zero of the derivative Airy function $\mathrm{Ai}^{\prime}\left[-\zeta^{\prime}\right]=0$. In the far field the Gaussian coupling window is accounted for by a convolution of the Hermite functions with a Gaussian (as discussed in Section 3). The asymmetry of the outermost peak of the Hermite functions, however, gives rise to an additional angular displacement in the final output beam, whereby we numerically find that

$$
\cos \bar{\alpha}_{j} \approx \frac{1}{k_{j} P \Theta_{m}}\left[\sqrt{2 p+1}-\frac{\zeta_{1}^{\prime}}{2^{1 / 2} p^{1 / 6}}\right]+\frac{1}{4}\left[\frac{\kappa}{P}+\frac{1}{P^{2} \Theta_{m}^{2}}\right]^{1 / 2} .
$$

Determination of the angular widths of the output beams follows by making the approximation $\Delta k_{y} / \Delta \beta_{j} \approx\left|\partial k_{y} / \partial \beta\right|_{\alpha=\bar{\alpha}_{j}, \beta=\bar{\beta}_{i}}$ (and similarly for $\Delta k_{z} / \Delta \alpha_{j}$ ). Within this approximation we find

$$
\begin{aligned}
& \Delta \alpha_{j}^{2}=\frac{k}{k_{j}^{2}}\left[\delta_{p, 0} \frac{n}{\sqrt{P R}}+\frac{\sqrt{n^{2}-1}}{P \sin ^{2} \bar{\alpha}_{j}}\right], \\
& \Delta \beta_{j}^{2}=\frac{k \sqrt{n^{2}-1}}{k_{j}^{2} R \sin ^{2} \bar{\alpha}_{j} \cos ^{2} \bar{\beta}_{j}} .
\end{aligned}
$$

Although similar in form to the expressions of Gorodetsky and Ilchenko, Eqs. (69), (73) and (74) possess slight differences due to differing definitions of the angles and the shape of resonators considered. Eq. (72) however differs from that of [36] due to the method of derivation, although we find Eq. (72) gives good numerical agreement with the positions of the peaks. We emphasis, however, that the output beam profiles shown in Figure 5(c) are further modulated by the Fresnel transmission coefficients. Due to the narrow width of the angular spectrum the associated variation of the transmission coefficients is small, however, if coupling to either ordinary or extraordinary propagating waves in the substrate is close to cut-off, then strong variation can occur resulting in so-called Fresnel filtering $[59,60]$ and a truncated output beam profile.

\section{CONCLUSIONS}

In this article we have theoretically studied the properties of WGMs in a $z$-cut uniaxial axisymmetric resonator placed in close proximity to a planar dielectric substrate, such as a prism. To do so, we first extended the approximate unperturbed mode profiles provided by Breunig et al. [43] to account for the open nature of the resonator and the vectorial nature of the underlying electromagnetic fields. Upon establishing an analytic angular spectrum representation of WGMs (Eq. (20)) we proceeded to study prism induced coupling to other WGMs and rigorously proved that coupling between modes of differing azimuthal order and parity was forbidden. Within a resonant mode approximation we subsequently derived expressions for the WGM profiles in the presence of a substrate, including reflection from and transmission through the interface (Eqs. (40), (42) and (45)). We have furthermore presented an analytic formalism capable of quantitatively predicting the resonance shifts and mode broadening that result from bringing a planar dielectric substrate from infinity into the evanescent field of a WGM (Eq. (68)). Our analysis was based on a generalisation of the Bethe-Schwinger 
perturbation equation which accounts for far-field radiative contributions and is hence suitable for application to open cavities [44]. Importantly, through use of generalised Fresnel reflection and transmission coefficients our theory can account for uniaxial substrates with an arbitrarily oriented optic axis and, moreover, is applicable to disc, toroidal and ellipsoidal shaped resonators, therefore extending alternative coupling theories found in the literature [37] to previously unconsidered geometries.

To complement our theoretical developments, extensive numerical examples were also presented. Supporting experimental results will be discussed in forthcoming publications. Competing red and blue shifts were found to dictate the total change in resonance frequency. The former red shift is well known and derives from the work done in polarising the dielectric substrate, however, the second less familiar effect originates from interference between the unperturbed and perturbed radiative component of the WGM in the far field, or a so-called radiative back action. Relative dominance of each effect was found to be influenced by the both the mode polarisation and the refractive index of the perturbing substrate relative to that of the resonator, since the latter determines whether WGMs couple to evanescent or propagating waves in the substrate. For both TE and TM WGMs, maximum red shifts occur when the refractive index of the perturbing substrate is approximately equal to that of the resonator, however, for larger substrate indices, the radiative contribution begins to dominate, leading not only to anomalous blue shifts, but also to significant mode broadening. An optimal regime, with respect to the substrate refractive index, in which maximum coupling can be achieved was also observed. Coupling of WGMs to the far field by means of a uniaxial prism, however, can manifest itself through two distinct channels, namely those of ordinary and extraordinary propagating waves. Orientation of the optic axis plays a central role in governing the relative losses between these channels. Exceptionally, when the resonator and substrate are fabricated from the same (birefringent) material this dependence renders polarisation-selective coupling with a high extinction ratio possible. Optimal selective coupling is found when the optic axis of the resonator and substrate are chosen to lie parallel to the resonator's symmetry axis (z-cut) and perpendicular to the substrate interface ( $x$-cut) respectively. Finally, we have discussed double refraction of a WGM into ordinary and extraordinary waves propagating in a nearby anisotropic substrate and moreover provided analytic formulae (Eqs. (69) and (72)-(74)) for their geometrical properties under general conditions.

The authors would like to acknowledge A. Aiello, Ch. Marquadt, U. Vogl, G. Schunk and D. V. Strekalov for helpful discussions. This work was funded by the Max Planck Society.

\section{A. REFLECTION AND TRANSMISSION AT A BIREFRIN- GENT INTERFACE}

In this appendix we derive the generalised Fresnel reflection and transmission coefficients of a plane wave in an isotropic medium incident upon a planar uniaxial interface whose surface normal is directed along $\hat{\mathbf{x}}$. The incident wave is assumed to have a complex wavevector, $\mathbf{k}=\left[k_{x}, k_{y}, k_{z}\right]$ (where $(x, y, z)$ is a fixed coordinate system as used in the main text), and can be decomposed into $\hat{\mathbf{s}}=(\mathbf{k} \times \hat{\mathbf{x}}) /|\mathbf{k} \times \hat{\mathbf{x}}|$ and $\hat{\mathbf{p}}=(\mathbf{k} \times \hat{\mathbf{s}}) /|\mathbf{k} \times \hat{\mathbf{s}}|$ polarised components which are perpendicular and parallel to the plane of incidence respectively. We further define the unit vectors $\hat{\mathbf{h}}=\hat{\mathbf{s}}$, which is parallel to the interface and perpendicular to the plane of incidence, and $\hat{\mathbf{g}}=\hat{\mathbf{x}} \times \hat{\mathbf{h}}$ which lies in the plane of incidence and is parallel to the interface. The incident wave gives rise to a reflected beam with wavevector $\mathbf{k}_{r}=\left[-k_{x}, k_{y}, k_{z}\right]$ which can be decomposed into $\hat{\mathbf{s}}_{r}=\hat{\mathbf{s}}$ and $\hat{\mathbf{p}}_{r}=\left[\mathbf{k}_{r} \times \hat{\mathbf{s}}\right] /\left|\mathbf{k}_{r} \times \hat{\mathbf{s}}\right|$ polarised components. Within a uniaxial medium, with arbitrary optic axis $\hat{\mathbf{c}}=\left[c_{x}, c_{y}, c_{z}\right]$, both ordinary and extraordinary wave components are generated with the associated unit polarisation vectors (corresponding to the electric field vector)

$$
\begin{aligned}
& \hat{\mathbf{o}}=\left(\mathbf{k}_{o} \times \hat{\mathbf{c}}\right) /\left|\mathbf{k}_{o} \times \hat{\mathbf{c}}\right| \\
& \hat{\mathbf{e}}=\stackrel{\leftrightarrow}{\epsilon}^{-1}\left(\mathbf{k}_{e} \times\left[\mathbf{k}_{e} \times \hat{\mathbf{c}}\right]\right) /\left|\overleftrightarrow{\epsilon}^{-1}\left(\mathbf{k}_{e} \times\left[\mathbf{k}_{e} \times \hat{\mathbf{c}}\right]\right)\right|
\end{aligned}
$$

where

$$
\stackrel{\leftrightarrow}{\epsilon}=\left(\begin{array}{ccc}
c_{x}^{2}\left(n_{e}^{2}-n_{o}^{2}\right)+n_{o}^{2} & c_{x} c_{y}\left(n_{e}^{2}-n_{o}^{2}\right) & c_{x} c_{z}\left(n_{e}^{2}-n_{o}^{2}\right) \\
c_{x} c_{y}\left(n_{e}^{2}-n_{o}^{2}\right) & c_{y}^{2}\left(n_{e}^{2}-n_{o}^{2}\right)+n_{o}^{2} & c_{y} c_{z}\left(n_{e}^{2}-n_{o}^{2}\right) \\
c_{x} c_{z}\left(n_{e}^{2}-n_{o}^{2}\right) & c_{y} c_{z}\left(n_{e}^{2}-n_{o}^{2}\right) & c_{z}^{2}\left(n_{e}^{2}-n_{o}^{2}\right)+n_{o}^{2}
\end{array}\right)
$$

is the permittivity tensor and $n_{0}\left(n_{e}\right)$ are the (extra)ordinary refractive indices of the dielectric substrate (note that in the main text these are denoted by $n_{o}^{\mathrm{sub}}$ and $n_{e}^{\mathrm{sub}}$ respectively). The corresponding wavevectors are $\mathbf{k}_{o}=\left[k_{0, x}, k_{y}, k_{z}\right]$ and $\mathbf{k}_{e}=$ $\left[k_{e, x}, k_{y}, k_{z}\right]$ where we note that the $k_{y}$ and $k_{z}$ components are conserved quantities across the interface (as per Snell's law). Letting $\hat{\mathbf{c}}=\left[\cos \chi_{c}, \sin \chi_{c} \cos \psi_{c}, \sin \chi_{c} \sin \psi_{c}\right]$, the $x$ components of the wavevectors are given by [76]

$$
\begin{aligned}
& k_{o, x}=\sqrt{n_{o}^{2} k^{2}-k_{y}^{2}-k_{z}^{2}} \\
& k_{e, x}=\left[v+\sqrt{v^{2}-4 u w}\right] /(2 u)
\end{aligned}
$$

and

$$
\begin{aligned}
u= & n_{e}^{-1} \sin ^{2} \chi_{c}+n_{o}^{-1} \cos ^{2} \chi_{c}, \\
v= & {\left[k_{y} \cos \psi_{c}+k_{z} \sin \psi_{c}\right]\left(n_{e}^{-2}+n_{o}^{-2}\right) \sin 2 \chi_{c}, } \\
w= & {\left[k_{y} \cos \psi_{c}+k_{z} \sin \psi_{c}\right]^{2}\left(n_{e}^{-2} \cos ^{2} \chi_{c}+n_{o}^{-2} \sin ^{2} \chi_{c}\right) } \\
& \quad+n_{e}^{-2}\left[-k_{y} \sin \psi_{c}+k_{z} \cos \psi_{c}\right]^{2}-k^{2} .
\end{aligned}
$$

The incident, reflected and transmitted electric fields can then be written (omitting the $\exp (-i \omega t)$ time dependence) as

$$
\begin{aligned}
& \mathbf{E}_{i}=\left[A_{s} \hat{\mathbf{s}}+A_{p} \hat{\mathbf{p}}\right] \exp [i \mathbf{k} \cdot \mathbf{r}] \\
& \mathbf{E}_{r}=\left[B_{s} \hat{\mathbf{s}}+B_{p} \hat{\mathbf{p}}_{r}\right] \exp \left[i \mathbf{k}_{r} \cdot \mathbf{r}\right] \\
& \mathbf{E}_{t}=C_{o} \hat{\mathbf{o}} \exp \left[i \mathbf{k}_{o} \cdot \mathbf{r}\right]+C_{e} \hat{\mathbf{e}} \exp \left[i \mathbf{k}_{e} \cdot \mathbf{r}\right],
\end{aligned}
$$

where $A_{s, p}, B_{s, p}, C_{o, e}$ are amplitude coefficients and $\omega$ is the optical frequency. From Maxwell's equation $i \omega \mu \mathbf{H}=\nabla \times \mathbf{E}$, we can also express the associated magnetic fields in the form:

$$
\begin{aligned}
\omega \mu \mathbf{H}_{i} & =\left[A_{S}(\mathbf{k} \times \hat{\mathbf{s}})+A_{p}(\mathbf{k} \times \hat{\mathbf{p}})\right] \exp [i \mathbf{k} \cdot \mathbf{r}] \\
\omega \mu \mathbf{H}_{r} & =\left[B_{S}\left(\mathbf{k}_{r} \times \hat{\mathbf{s}}\right)+B_{p}\left(\mathbf{k}_{r} \times \hat{\mathbf{p}}_{r}\right)\right] \exp \left[i \mathbf{k}_{r} \cdot \mathbf{r}\right] \\
\omega \mu \mathbf{H}_{t} & =C_{o}\left(\mathbf{k}_{o} \times \hat{\mathbf{o}}\right) \exp \left[i \mathbf{k}_{o} \cdot \mathbf{r}\right]+C_{e}\left(\mathbf{k}_{e} \times \hat{\mathbf{e}}\right) \exp \left[i \mathbf{k}_{e} \cdot \mathbf{r}\right] .
\end{aligned}
$$

Enforcing continuity of the components of the electric and magnetic fields tangential to the interface (i.e. $\mathbf{E} \cdot \hat{\mathbf{g}}^{*}, \mathbf{E} \cdot \hat{\mathbf{h}}^{*}, \mathbf{H} \cdot \hat{\mathbf{g}}^{*}$ and $\mathbf{H} \cdot \hat{\mathbf{h}}^{*}$ ) yields four equations viz.

$$
\begin{aligned}
A_{s}+B_{s} & =C_{o} \hat{\mathbf{o}} \cdot \hat{\mathbf{h}}^{*}+C_{e} \hat{\mathbf{e}} \cdot \hat{\mathbf{h}}^{*} \\
k_{x}\left(A_{s}-B_{s}\right) & =C_{o}\left(\mathbf{k}_{o} \times \hat{\mathbf{o}}\right) \cdot \hat{\mathbf{g}}^{*}+C_{e}\left(\mathbf{k}_{e} \times \hat{\mathbf{e}}\right) \cdot \hat{\mathbf{g}}^{*} \\
k_{x}\left(A_{p}-B_{p}\right) & =C_{o} \hat{\mathbf{o}} \cdot \hat{\mathbf{g}}^{*}+C_{e} \hat{\mathbf{e}} \cdot \hat{\mathbf{g}}^{*} \\
-A_{p}-B_{p} & =C_{o}\left(\mathbf{k}_{o} \times \hat{\mathbf{o}}\right) \cdot \hat{\mathbf{h}}^{*}+C_{e}\left(\mathbf{k}_{e} \times \hat{\mathbf{e}}\right) \cdot \hat{\mathbf{h}}^{*}
\end{aligned}
$$


where $k_{x}=\mathbf{k} \cdot \hat{\mathbf{x}}$ is the $x$ component of $\mathbf{k}$. We can define generalised reflection and transmission coefficients according to [77]

$$
\begin{array}{ll}
B_{s}=r_{s s} A_{s}+r_{p s} A_{p} \\
C_{o}=t_{s o} A_{s}+t_{p o} A_{p}
\end{array} \quad, \quad \begin{aligned}
& B_{p}=r_{s p} A_{s}+r_{p p} A_{p} \\
& C_{e}=t_{s e} A_{s}+t_{p e} A_{p}
\end{aligned}
$$

which can be found by solving Eqs. (88)-(91) yielding

$$
\begin{aligned}
r_{s s} & =\frac{M_{-}^{o} N_{-}^{e}-M_{-}^{e} N_{-}^{o}}{M_{+}^{o} N_{-}^{e}-M_{+}^{e} N_{-}^{o}}, r_{p p}=\frac{M_{+}^{o} N_{+}^{e}-M_{+}^{e} N_{+}^{o}}{M_{+}^{o} N_{-}^{e}-M_{+}^{e} N_{-}^{o}} \\
r_{p s} & =\frac{M_{+}^{o} M_{-}^{e}-M_{-}^{o} M_{+}^{e}}{M_{+}^{o} N_{-}^{e}-M_{+}^{e} N_{-}^{o}}, r_{s p}=\frac{N_{-}^{o} N_{+}^{e}-N_{+}^{o} N_{-}^{e}}{M_{+}^{o} N_{-}^{e}-M_{+}^{e} N_{-}^{o}} \\
t_{s o} & =\frac{2 N_{-}^{e}}{M_{+}^{o} N_{-}^{e}-M_{+}^{e} N_{-}^{o}}, t_{s e}=\frac{-2 N_{-}^{o}}{M_{+}^{o} N_{-}^{e}-M_{+}^{e} N_{-}^{o}} \\
t_{p o} & =\frac{-2 M_{+}^{e}}{M_{+}^{o} N_{-}^{e}-M_{+}^{e} N_{-}^{o}} \quad, \quad t_{p e}=\frac{2 M_{+}^{o}}{M_{+}^{o} N_{-}^{e}-M_{+}^{e} N_{-}^{o}}
\end{aligned}
$$

and

$$
\begin{aligned}
M_{ \pm}^{o} & =\hat{\mathbf{o}} \cdot \hat{\mathbf{h}}^{*} \pm\left(\mathbf{k}_{o} \times \hat{\mathbf{o}}\right) \cdot \hat{\mathbf{g}}^{*} / k_{x} \\
M_{ \pm}^{e} & =\hat{\mathbf{e}} \cdot \hat{\mathbf{h}}^{*} \pm\left(\mathbf{k}_{e} \times \hat{\mathbf{g}}\right) \cdot \hat{\mathbf{g}}^{*} / k_{x} \\
N_{ \pm}^{o} & =-\left(\mathbf{k}_{o} \times \hat{\mathbf{o}}\right) \cdot \hat{\mathbf{h}}^{*} / k \pm k \hat{\mathbf{o}} \cdot \hat{\mathbf{g}}^{*} / k_{x} \\
N_{ \pm}^{e} & =-\left(\mathbf{k}_{e} \times \hat{\mathbf{e}}\right) \cdot \hat{\mathbf{h}}^{*} / k \pm k \hat{\mathbf{e}} \cdot \hat{\mathbf{g}}^{*} / k_{x} .
\end{aligned}
$$

We note that Eqs. (94)-(101) are equivalent to those presented in [76] for propagating waves, however differ for evanescent waves since we normalise unit vectors such that $\mathbf{u} \cdot \mathbf{u}^{*}=1$ as opposed to $\mathbf{u} \cdot \mathbf{u}=1$. We elect to use this normalisation convention since it maintains the physical definition of the Fresnel coefficients as the amplitude ratio of the respective field component [78].

\section{B. BREIT-WIGNER LINE SHAPE WITH MATERIAL AB- SORPTION}

We start by considering the scattering amplitude of a given WGM which near resonance takes the form

$$
\eta_{\mathbf{v}}(\omega)=-\frac{\gamma_{\mathbf{v}, \mathrm{rad}} / 2}{\gamma_{\mathbf{v}, \mathrm{rad}} / 2-i\left(\omega-\omega_{\mathbf{v}}\right)} \triangleq-\frac{1}{1-i \beta(\omega)}
$$

when absorption in the resonator is neglected. To account for the effect of absorption we must consider the dependence of $\beta(\omega)$ on the complex refractive index $\bar{n}=n+i \kappa$. Following [57] we perform a Taylor expansion of $\beta(\omega, \bar{n})$ around the resonance frequency $\omega_{\mathbf{v}}$ and the real part of the refractive index $n$ whereby

$$
\beta(\omega) \approx\left(\omega-\omega_{\mathbf{v}}\right) \partial_{\omega} \beta\left(\omega_{\mathbf{v}}, n\right)+i \kappa \partial_{\bar{n}} \beta\left(\omega_{\mathbf{v}}, n\right)
$$

and where $\beta\left(\omega_{\mathbf{v}}, n\right)=0$ has been used. Substituting Eq. (103) into Eq. (102) yields

$$
\eta_{\mathbf{v}}(\omega) \approx-\frac{1}{1+\kappa \partial_{\bar{n}} \beta\left(\omega_{\mathbf{v}}, n\right)-i\left(\omega-\omega_{\mathbf{v}}\right) \partial_{\omega} \beta\left(\omega_{\mathbf{v}}, n\right)} .
$$

Noting $\partial_{\omega} \beta\left(\omega_{\mathbf{v}}, n\right)=2 / \gamma_{\mathbf{v}, \text { rad }}$, we observe that Eq. (104) also possesses a Breit-Wigner line shape, albeit with a modified line width of $\gamma_{\text {tot }}=\gamma_{\mathbf{v} \text {,rad }}\left(1+\kappa \partial_{\bar{n}} \beta\left(\omega_{\mathbf{v}}, n\right)\right)=\gamma_{\mathbf{v}, \text { rad }}+\gamma_{\mathbf{v}, \text { abs }}$. We can hence deduce $\kappa \partial_{\bar{n}} \beta\left(\omega_{\mathbf{v}}, n\right)=\gamma_{\mathbf{v}, \text { abs }} / \gamma_{\mathbf{v} \text {,rad }}$ whereby Eq. (104) yields Eq. (38) of the main text.

\section{ANISOTROPIC BETHE-SCHWINGER EQUATION}

The eigenmodes of an open system can be written in the form $\overline{\mathbf{E}}_{\mathbf{v}}(\mathbf{r}, t)=\mathbf{E}_{\mathbf{v}}(\mathbf{r}) \exp [-i \bar{\omega} t]$, where $\bar{\omega}=\omega-i \gamma / 2$ is the complex eigenfrequency which describes both the real resonance frequency $\omega$ and the resonance lifetime $1 / \gamma$. The associated magnetic field $\overline{\mathbf{H}}_{\mathbf{v}}(\mathbf{r}, t)$ can be similarly defined. The eigenmodes must satisfy Maxwell's equations, which allowing for anisotropic media reduce to

$$
\nabla \times \mathbf{E}_{\mathbf{v}}=i \bar{\omega} \mathbf{B}_{\mathbf{v}} \text { and } \nabla \times \mathbf{H}_{\mathbf{v}}=-i \bar{\omega} \mathbf{D}_{\mathbf{v}}
$$

where $\mathbf{D}=\overleftrightarrow{\epsilon} \mathbf{E}, \mathbf{B}=\overleftrightarrow{\mu} \mathbf{H}$, and $\overleftrightarrow{\epsilon}=\overleftrightarrow{\epsilon}(\mathbf{r})$ and $\overleftrightarrow{\mu}=\overleftrightarrow{\mu}(\mathbf{r})$ are the electric permittivity and magnetic permeability tensors describing the cavity and its surroundings. Modification of the dielectric environment results in a modified set of eigenmodes $\overline{\mathbf{E}}_{\mathbf{v}}^{\prime}(\mathbf{r}, t)=\mathbf{E}_{\mathbf{v}}^{\prime}(\mathbf{r}) \exp \left[-i \bar{\omega}^{\prime} t\right]$ (and similarly for $\overline{\mathbf{H}}_{\mathbf{v}}^{\prime}$ ), which must also satisfy an analogous set of equations to Eq. (105) with the replacements $\mathbf{E}_{\mathbf{v}} \rightarrow \mathbf{E}_{\mathbf{v}}^{\prime}, \mathbf{H}_{\mathbf{v}} \rightarrow \mathbf{H}_{\mathbf{v}}^{\prime}, \overleftrightarrow{\epsilon}(\mathbf{r}) \rightarrow \overleftrightarrow{\epsilon}^{\prime}(\mathbf{r})$ and $\overleftrightarrow{\mu}(\mathbf{r}) \rightarrow \overleftrightarrow{\mu}^{\prime}(\mathbf{r})$. Subtracting these sets of equations yields

$$
\begin{aligned}
\nabla \times \delta \mathbf{E}_{\mathbf{v}} & =i \bar{\omega}^{\prime} \mathbf{B}_{\mathbf{v}}^{\prime}-i \bar{\omega} \mathbf{B}_{\mathbf{v}} \\
\nabla \times \delta \mathbf{H}_{\mathbf{v}} & =-i \bar{\omega}^{\prime} \mathbf{D}_{\mathbf{v}}^{\prime}+i \bar{\omega} \mathbf{D}_{\mathbf{v}}
\end{aligned}
$$

where $\delta \mathbf{E}_{\mathbf{v}}=\mathbf{E}_{\mathbf{v}}^{\prime}-\mathbf{E}_{\mathbf{v}}$ (and similarly for $\delta \mathbf{H}_{\mathbf{v}}$ ). Now forming the inner products $\mathbf{H}_{\mathbf{v}}^{*} \cdot \nabla \times \delta \mathbf{E}_{\mathbf{v}}$ and $\mathbf{E}_{\mathbf{v}}^{*} \cdot \nabla \times \delta \mathbf{H}_{\mathbf{v}}$, using the vector identity $\mathbf{A} \cdot(\nabla \times \mathbf{B})=\mathbf{B} \cdot(\nabla \times \mathbf{A})-\nabla \cdot(\mathbf{A} \times \mathbf{B})$ and subtracting the resulting expressions we obtain

$$
\begin{array}{r}
i \bar{\omega}^{*}\left(\delta \mathbf{E}_{\mathbf{v}} \cdot \mathbf{D}_{\mathbf{v}}^{*}+\delta \mathbf{H}_{\mathbf{v}} \cdot \mathbf{B}_{\mathbf{v}}^{*}\right)-\nabla \cdot\left(\mathbf{H}_{\mathbf{v}}^{*} \times \delta \mathbf{E}_{\mathbf{v}}-\mathbf{E}_{\mathbf{v}}^{*} \times \delta \mathbf{H}_{\mathbf{v}}\right) \\
=i \bar{\omega}^{\prime}\left(\mathbf{E}_{\mathbf{v}}^{*} \cdot \mathbf{D}_{\mathbf{v}}^{\prime}+\mathbf{H}_{\mathbf{v}}^{*} \cdot \mathbf{B}_{\mathbf{v}}^{\prime}\right)-i \bar{\omega}\left(\mathbf{E}_{\mathbf{v}}^{*} \cdot \mathbf{D}_{\mathbf{v}}+\mathbf{H}_{\mathbf{v}}^{*} \cdot \mathbf{B}_{\mathbf{v}}\right) .
\end{array}
$$

Integrating over a spherical volume of radius $R_{V}$ and use of Gauss's theorem gives Eq. (46) in the main text from which both the resonance shift $\delta \omega=\omega^{\prime}-\omega$ and mode broadening $\delta \gamma=\gamma^{\prime}-\gamma$ can be extracted by rewriting Eq. (46) as

$$
\begin{aligned}
\delta \bar{\omega}=[- & (\delta \bar{\omega}+\bar{\omega}) N_{\mathbf{v}}^{(1)}-i N_{\mathbf{v}}^{(2)} \\
& \left.+\bar{\omega}^{*} N_{\mathbf{v}}^{(3)}+\bar{\omega}\left(N_{\mathbf{v}}^{(4)}-N_{\mathbf{v}}^{(0)}\right)\right] / N_{\mathbf{v}}^{(0)},
\end{aligned}
$$

where we have let $\overleftrightarrow{\epsilon}^{\prime}(\mathbf{r})=\overleftrightarrow{\epsilon}(\mathbf{r})+\delta \overleftrightarrow{\epsilon}(\mathbf{r})$ (and similarly for $\mu^{\prime}$ ) and have defined the integral terms

$$
\begin{aligned}
& N_{\mathbf{v}}^{(0)}=\int_{V} \mathbf{E}_{\mathbf{v}}^{*} \overleftrightarrow{\epsilon} \mathbf{E}_{\mathbf{v}}^{\prime}+\mathbf{H}_{\mathbf{v}}^{*} \overleftrightarrow{\mu} \mathbf{H}_{\mathbf{v}}^{\prime} d V \\
& N_{\mathbf{v}}^{(1)}=\int_{V} \mathbf{E}_{\mathbf{v}}^{*} \delta \overleftrightarrow{\epsilon} \mathbf{E}_{\mathbf{v}}^{\prime}+\mathbf{H}_{\mathbf{v}}^{*} \delta \overleftrightarrow{\mu} \mathbf{H}_{\mathbf{v}}^{\prime} d V \\
& N_{\mathbf{v}}^{(2)}=\oint_{S}\left(\delta \mathbf{E}_{\mathbf{v}} \times \mathbf{H}_{\mathbf{v}}^{*}+\mathbf{E}_{\mathbf{v}}^{*} \times \delta \mathbf{H}_{\mathbf{v}}\right) \cdot d \mathbf{S} \\
& N_{\mathbf{v}}^{(3)}=\int_{V} \delta \mathbf{E}_{\mathbf{v}} \stackrel{\leftrightarrow}{\epsilon} \mathbf{E}_{\mathbf{v}}^{*}+\delta \mathbf{H}_{\mathbf{v}} \overleftrightarrow{\mu} \mathbf{H}_{\mathbf{v}}^{*} d V \\
& N_{\mathbf{v}}^{(4)}=\int_{V} \mathbf{E}_{\mathbf{v}}^{*} \overleftrightarrow{\epsilon} \mathbf{E}_{\mathbf{v}}+\delta \mathbf{H}_{\mathbf{v}}^{*} \overleftrightarrow{\mu} \mathbf{H}_{\mathbf{v}} d V .
\end{aligned}
$$

After application of Gauss's theorem we let $R_{V} \rightarrow \infty$, such that the surface integral in $N_{\mathbf{v}}^{(2)}$ is taken at infinity as discussed in the main text.

Practical WGM resonators are typically fabricated from materials for which the imaginary part of the permittivity is small. Although absorption can still play an important role in determining the line width of WGMs it is nevertheless safe to assume that $\epsilon$ is real when evaluating the integrals in Eqs. (110)-(114). Noting that the electric permittivity tensor $\epsilon$ is also symmetric and 
assuming $\bar{\omega} \approx \bar{\omega}^{*}$, i.e. restricting attention to high $Q$ resonances it then follows that $\bar{\omega}^{*} N_{\mathbf{v}}^{(3)}+\bar{\omega}\left[N_{\mathbf{v}}^{(4)}-N_{\mathbf{v}}^{(0)}\right]=0$. Making the further assumption that $\delta \omega \ll \omega$ and $\delta \gamma \ll \omega$ Eq. (46) simplifies to Eq. (47). Validity conditions of the Bethe-Schwinger equation are also discussed in Refs. [44] and [61] .

\section{REFERENCES}

1. V. Sandoghdar, F. Treussart, J. Hare, V. Lefèvre-Seguin, J. M. Raimond, and S. Haroche, "Very low threshold whispering-gallery-mode microsphere laser," Phys. Rev. A 54, R1777-R1780 (1996).

2. C. Gmachl, F. Capasso, E. E. Narimanov, J. U. Nöckel, A. D. Stone, J. Faist, D. L. Sivco, and A. Y. Cho, "High-Power Directional Emission from Microlasers with Chaotic Resonators," Science 5, 1556-1564 (1998).

3. S. M. Spillane, T. J. Kippenberg, and K. J. Vahala, "Ultralow-threshold Raman laser using a spherical dielectric microcavity," Nature 415, 621623 (2002).

4. J. U. Fürst, D. V. Strekalov, D. Elser, A. Aiello, U. L. Andersen, C. Marquardt, and G. Leuchs, "Quantum Light from a Whispering-Gallery-Mode Disk Resonator," Phys. Rev. Lett. 106, 113901 (2011).

5. G. Gagliardi and H.-P. Loock, Cavity-Enhanced Spectroscopy and Sensing, vol. 179 of Springer Series in Optical Sciences (Springer-Verlag, Berlin, 2014).

6. A. Savchenkov, A. B. Matsko, V. S. Ilchenko, I. Solomatine, D. Seidel, and L. Maleki, "Tunable Optical Frequency Comb with a Crystalline Whispering Gallery Mode Resonator," Phys. Rev. Lett. 101, 093902 (2008).

7. T. J. Kippenberg, R. Holzwarth, and S. A. Diddams, "Microresonatorbased optical frequency combs," Science 332, 555-9 (2011).

8. D. W. Vernooy, A. Furusawa, N. P. Georgiades, V. S. Ilchenko, and H. J. Kimble, "Cavity QED with high-Q whispering gallery modes," Phys. Rev. A 57, R2293-R2296 (1998).

9. T. Aoki, B. Dayan, E. Wilcut, W. P. Bowen, A. S. Parkins, T. J. Kippenberg, K. J. Vahala, and H. J. Kimble, "Observation of strong coupling between one atom and a monolithic microresonator," Nature 443, 671-674 (2006).

10. M. R. Foreman, J. D. Swaim, and F. Vollmer, "Whispering gallery mode sensors," Adv. Opt. Photon. 7, 168-240 (2015).

11. V. S. Ilchenko, A. A. Savchenkov, A. B. Matsko, and L. Maleki, "Nonlinear optics and crystalline whispering gallery mode cavities." Phys. Rev. Lett. 92, 043903 (2004).

12. D. V. Strekalov, C. Marquardt, A. B. Matsko, H. G. L. Schwefel, and G. Leuchs, "Nonlinear and Quantum Optics with Whispering Gallery Resonators," arXiv (2016).

13. A. B. Matsko, A. A. Savchenkov, V. S. Ilchenko, D. Seidel, and L. Maleki, "Optomechanics with surface-acoustic-wave whispering-gallery modes," Phys. Rev. Lett. 103, 257403 (2009).

14. A. Schliesser and T. J. Kippenberg, "Cavity optomechanics with whispering-gallery-mode optical micro-resonators," in "Advances In Atomic, Molecular, and Optical Physics," , vol. 58, P. Berman, E. Arimondo, and C. Lin, eds. (Academic Press, New York, 2010), pp. 207323.

15. F. Monifi, S. K. Özdemir, and L. Yang, "Tunable add-drop filter using an active whispering gallery mode microcavity," Appl. Phys. Lett. 103, 181103 (2013).

16. G. Schunk, U. Vogl, D. V. Strekalov, M. Förtsch, F. Sedlmeir, H. G. L. Schwefel, M. Göbelt, S. Christiansen, G. Leuchs, and C. Marquardt, "Interfacing transitions of different alkali atoms and telecom bands using one narrowband photon pair source," Optica 2, 773-778 (2015).

17. H. J. Kimble, "The quantum internet," Nature 453, 1023-1030 (2008).

18. D. N. Matsukevich and A. Kuzmich, "Quantum state transfer between matter and light," Science 306, 663-666 (2004).

19. A. Y. Smirnov, S. N. Rashkeev, and A. M. Zagoskin, "Polarization switching in optical microsphere resonator," Appl. Phys. Lett. 80, 35033505 (2002).

20. I. Breunig, "Three-wave mixing in whispering gallery resonators," Laser Photon. Rev. 10, 681-687 (2016).

21. B. Sturman and I. Breunig, "Generic description of second-order non- linear phenomena in whispering-gallery resonators," J. Opt. Soc. Am. B 28, 2465-2471 (2011).

22. A. A. Savchenkov, V. S. Ilchenko, A. B. Matsko, and L. Maleki, "Tunable filter based on whispering gallery modes," Electron. Letters 39, 389-391 (2003).

23. D. Armani, B. Min, A. Martin, and K. J. Vahala, "Electrical thermooptic tuning of ultrahigh-Q microtoroid resonators," Appl. Phys. Lett. 85, 5439-5441 (2004).

24. J. M. Ward and S. N. Chormaic, "Thermo-optical tuning of whispering gallery modes in Er:Yb co-doped phosphate glass microspheres," Appl. Phys. B 100, 847-850 (2010).

25. W. Lin, H. Zhang, B. Liu, B. Song, Y. Li, C. Yang, and Y. Liu, "Lasertuned whispering gallery modes in a solid-core microstructured optical fibre integrated with magnetic fluids," Sci. Rep. 5, 17791 (2015).

26. W. von Klitzing, R. Long, V. S. Ilchenko, J. Hare, and V. Lefèvre-Seguin, "Frequency tuning of the whispering-gallery modes of silica microspheres for cavity quantum electrodynamics and spectroscopy." Opt. Lett. 26, 166-168 (2001).

27. M. Pöllinger, D. O'Shea, F. Warken, and A. Rauschenbeutel, "UltrahighQ tunable whispering-gallery-mode microresonator," Phys. Rev. Lett. 103, 053901 (2009).

28. R. Madugani, Y. Yang, J. M. Ward, J. D. Riordan, S. Coppola, V. Vespini, S. Grilli, A. Finizio, P. Ferraro, and S. Nic Chormaic, "Terahertz tuning of whispering gallery modes in a PDMS stand-alone, stretchable microsphere." Opt. Lett. 37, 4762-4 (2012).

29. T. Ioppolo and M. V. Ötügen, "Pressure tuning of whispering gallery mode resonators," J. Opt. Soc. Am B 24, 2721-2726 (2007).

30. R. Henze, T. Seifert, J. M. Ward, and O. Benson, "Tuning whispering gallery modes using internal aerostatic pressure." Opt. Lett. 36, 4536-8 (2011).

31. G. Schunk, U. Vogl, F. Sedlmeir, D. V. Strekalov, A. Otterpohl, V. Averchenko, H. G. L. Schwefel, G. Leuchs, and C. Marquardt, "Frequency tuning of single photons from a whisperinggallery mode resonator to $\mathrm{MHz}$-wide transitions," J. Mod. Opt. doi:10.1080/09500340.2016.1148211 (2016).

32. J. Wiersig, "Boundary element method for resonances in dielectric microcavities," J. Opt. A: Pure Appl. Opt. 5, 53-60 (2003).

33. I. Teraoka and S. Arnold, "Theory of resonance shifts in TE and TM whispering gallery modes by nonradial perturbations for sensing applications," J. Opt. Soc. Am. B 23, 1381-1389 (2006).

34. A. Kaplan, M. Tomes, T. Carmon, M. Kozlov, O. Cohen, and H. G. L. Schwefel, "Finite element simulation of a perturbed axial- symmetric whispering-gallery mode and its use for intensity enhancement with a nanoparticle coupled to a microtoroid," Opt. Express 21, 14169-14180 (2013).

35. M. R. Foreman and F. Vollmer, "Theory of resonance shifts of whispering gallery modes by arbitrary plasmonic nanoparticles," New J. Phys 15, 083006 (2013).

36. M. L. Gorodetsky and V. S. Ilchenko, "High-Q optical whispering-gallery microresonators : precession approach for spherical mode analysis and emission patterns with prism couplers," Opt. Commun. 113, 133-143 (1994).

37. M. L. Gorodetsky and V. S. Ilchenko, "Optical microsphere resonators : optimal coupling to high-Q whispering-gallery modes," J. Opt. Soc. Am. B 16, 147-154 (1999).

38. M. A. C. Shirazi, W. Yu, S. Vincent, and T. Lu, "Cylindrical beam propagation modelling of perturbed whispering-gallery mode microcavities," Opt. Express 21, 30243-30254 (2013).

39. X. Du, S. Vincent, and T. Lu, "Full-vectorial whispering-gallery-mode cavity analysis," Opt. Express 21, 22012 (2013)

40. X. Du, S. Vincent, M. Faucher, M.-J. Picard, and T. Lu, "Generalized fullvector multi-mode matching analysis of whispering gallery microcavities." Opt. Express 22, 13507-14 (2014).

41. J. A. Haigh, S. Langenfeld, N. J. Lambert, J. J. Baumberg, A. J. Ramsay, A. Nunnenkamp, and A. J. Ferguson, "Magneto-optical coupling in whispering-gallery-mode resonators," Phys. Rev. A 92, 063845 (2015).

42. F. Sedlmeir, M. R. Foreman, R. Zeltner, U. Vogl, G. Schunk, C. Marquardt, G. Leuchs, and H. G. L. Schwefel, "Polarization selective out- 
coupling of whispering gallery modes," in preparation (2016).

43. I. Breunig, B. Sturman, F. Sedlmeir, H. G. L. Schwefel, and K. Buse, "Whispering gallery modes at the rim of an axisymmetric optical resonator: analytical versus numerical description and comparison with experiment." Opt. Express 21, 30683-30692 (2013).

44. F. Ruesink, H. M. Doeleman, R. Hendrikx, A. F. Koenderink, and E. Verhagen, "Perturbing open cavities: Anomalous resonance frequency shifts in a hybrid cavity-nanoantenna system," Phys. Rev. Lett. 115, 203904 (2015).

45. M. Ornigotti and A. Aiello, "Analytical Approximations of Whispering Gallery Modes in Anisotropic Ellipsoidal Resonators," Phys. Res. Int. 2014, 615605 (2014)

46. Y. A. Demchenko and M. L. Gorodetsky, "Analytical estimates of eigenfrequencies, dispersion, and field distribution in whispering gallery resonators," J. Opt. Soc. Am. B 30, 3056-3063 (2013).

47. C. Junge, D. O'Shea, J. Volz, and A. Rauschenbeutel, "Strong Coupling between Single Atoms and Nontransversal Photons," Phys. Rev. Lett. 110, 213604 (2013).

48. M. R. Foreman, S. Avino, R. Zullo, H.-P. Loock, F. Vollmer, and G. Gagliardi, "Enhanced nanoparticle detection with liquid droplet resonators," Eur. Phys. J. Spec. Top. 223, 1971-1988 (2014).

49. M. Oxborrow, "Traceable 2-D Finite-Element Simulation of the Whispering-Gallery Modes of Axisymmetric Electromagnetic Resonators," IEEE T Microwav. Theo. Tech. 55, 1209-1218 (2007).

50. I. Teraoka and S. Arnold, "Whispering-gallery modes in a microsphere coated with a high-refractive index layer : polarization-dependent sensitivity enhancement of the resonance-shift sensor and TE-TM resonance matching," J. Opt. Soc. Am. B 24, 653-659 (2007).

51. M. R. Foreman and F. Vollmer, "Optical Tracking of Anomalous Diffusion Kinetics in Polymer Microspheres," Phys. Rev. Lett. 114, 118001 (2015).

52. B. R. Johnson, "Morphology-dependent resonances of a dielectric sphere on a conducting plane," J. Opt. Soc. Am. A 11, 2055-2064 (1994).

53. B. R. Johnson, "Calculation of light scattering from a spherical particle on a surface by the multipole expansion method," J. Opt. Soc. Am. A 13, 326-337 (1996).

54. C. F. Bohren and Huffman D R, Absorption and scattering of light by small particles (John Wiley \& Sons, Ltd., New York, 1983).

55. K. McVoy, L. Heller, and M. Bolsterli, "Optical Analysis of Potential Well Resonances," Rev. Mod. Phys. 39, 245-258 (1967).

56. A. Erdelyi, W. Magnus, F. Oberhettinger, and F. G. Tricomi, Tables of integral transforms: Volume II (McGraw Hill, New York, 1954).

57. B. R. Johnson, "Theory of morphology-dependent resonances: shape resonances and width formulas," J. Opt. Soc. Am. A 10, 343-352 (1993).

58. M. L. Gorodetsky, A. A. Savchenkov, and V. S. Ilchenko, "Ultimate Q of optical microsphere resonators," Opt. Lett. 21, 453-455 (1996).

59. N. B. Rex, H. E. Tureci, H. G. L. Schwefel, R. K. Chang, A. Douglas Stone, and A. D. Stone, "Fresnel Filtering in Lasing Emission from Scarred Modes of Wave-Chaotic Optical Resonators," Phys. Rev. Lett. 88, 094102 (2002).
60. H. E. Tureci and A. D. Stone, "Deviation from Snell's law for beams transmitted near the critical angle: application to microcavity lasers," Opt. Lett. 27, 7-9 (2002).

61. R. A. Waldron, "Perturbation theory of resonant cavities," Proc. Inst. Electr. Eng. 107, 272-274 (1960).

62. L. Mandel and E. Wolf, Optical Coherence and Quantum Optics (Cambridge University Press, Cambridge, 1995).

63. D. E. Zelmon, D. L. Small, and D. Jundt, "Infrared corrected Sellmeier coefficients for congruently grown lithium niobate and $5 \mathrm{~mol} . \%$ magnesium oxide-doped lithium niobate," J. Opt. Soc. Am. B 14, 3319-3322 (1997).

64. G. Ghosh, "Dispersion-equation coefficients for the refractive index and birefringence of calcite and quartz crystals," Opt. Commun. 163, 95-102 (1999).

65. M. J. Dodge, "Refractive properties of magnesium fluoride," Appl. Opt. 23, 1980-1985 (1984).

66. D. Eimerl, L. Davis, S. Velsko, E. K. Graham, and A. Zalkin, "Optical, mechanical, and thermal properties of barium borate," J. Appl. Phys. 62, 1968-1983 (1987).

67. T. Sugawara, R. Komatsu, and S. Uda, "Linear and nonlinear optical properties of lithium tetraborate," Solid State Commun. 107, 233-237 (1998).

68. M. A. Jeppesen, "Some Optical, Thermo-Optical, and Piezo-Optical Properties of Synthetic Sapphire," J. Opt. Soc. Am. 48, 629 (1958).

69. J. R. Devore, "Refractive Indices of Rutile and Sphalerite," J. Opt. Soc. Am. 41, 416 (1951).

70. W. L. Bond, "Measurement of the refractive indices of several crystals," J. Appl. Phys. 36, 1674-1677 (1965).

71. A. S. Barker and M. llegems, "Infrared lattice vibrations and freeelectron dispersion in GaN," Phys. Rev. B 7, 743-750 (1973).

72. V. E. Babicheva and A. V. Lavrinenko, "A plasmonic modulator based on a metal-insulator-metal waveguide with a barium titanate core," Photon. Lett. Pol. 5, 57-59 (2013)

73. K. F. Hulme, O. Jones, P. H. Davies, and M. V. Hobden, "Synthetic proustite $\left(\mathrm{Ag}_{3} \mathrm{AsS}_{3}\right)$ : A new crystal for optical mixing," Appl. Phys. Lett. 10, 133-135 (1967)

74. S. Das, G. C. Bhar, S. Gangopadhyay, and C. Ghosh, "Linear and nonlinear optical properties of $\mathrm{ZnGeP}_{2}$ crystal for infrared laser device applications: revisited" Appl. Opt. 42, 4335-4340 (2003).

75. G. Schunk, J. U. Fürst, M. Förtsch, D. V. Strekalov, U. Vogl, F. Sedlmeir, H. G. L. Schwefel, G. Leuchs, and C. Marquardt, "Identifying modes of large whispering-gallery mode resonators from the spectrum and emission pattern," Opt. Express 22, 30795-30806 (2014).

76. C. Gu and P. Yeh, "Extended Jones matrix method. II," J. Opt. Soc. Am. A 10, 966-973 (1993).

77. P. Yeh, "Electromagnetic propagation in birefringent layered media," J. Opt. Soc. Am 69, 742-756 (1979).

78. A. Norrman, T. Setälä, and A. T. Friberg, "Partial spatial coherence and partial polarization in random evanescent fields on lossless interfaces." J. Opt. Soc. Am. A 28, 391-400 (2011). 